\title{
A Quantitative Data-Driven Analysis (QDA) Framework for Resting-state fMRI: a Study of the Impact of Adult Age
}

\author{
Xia Li ${ }^{1}$, Håkan Fischer ${ }^{2,3}$, Amirhossein Manzouri², Kristoffer N.T. Månsson ${ }^{2}$, \\ Tie-Qiang $\mathrm{Li}^{1,4,5^{*}}$, \\ ${ }^{1}$ Institute of Informatics Engineering, China Jiliang University, Hangzhou, China \\ 2Department of Psychology, Stockholm University, Sweden \\ ${ }^{3}$ Stockholm University Brain Imaging Centre, Sweden \\ ${ }^{4}$ Department of Clinical Science, Intervention, and Technology, Karolinska Institute, Sweden \\ ${ }^{5}$ Department of Medical Radiation and Nuclear Medicine, Karolinska University Hospital, Sweden
}

*The corresponding author

Tie-Qiang Li

Department of Clinical Science, Intervention, and Technology,

Karolinska Institutet, Sweden

Email: tie-qiang.li@ki.se

Tel: +46707374215 


\begin{abstract}
:
Purpose: The objective of this study is to introduce a new quantitative data-driven analysis (QDA) framework for the analysis of resting-state fMRI (R-fMRI) and use it to investigate the effect of adult age on resting-state functional connectivity (RFC).

Methods Whole-brain R-fMRI measurements were conducted on a 3T clinical MRI scanner in 227 healthy adult volunteers $(\mathrm{N}=227$, aged 18-74 years old, male/female=99/128). With the proposed QDA framework we derived two types of voxel-wise RFC metrics: the connectivity strength index (CSI) and connectivity density index (CDI) utilizing the convolutions of the cross-correlation (CC) histogram with different kernels. Furthermore, we assessed the negative and positive portions of these metrics separately.
\end{abstract}

Results: With the QDA framework we found age-related declines of RFC metrics in the superior and middle frontal gyrus (MFG), posterior cingulate cortex (PCC), right insula and inferior parietal lobule (IPL) of the default mode network (DMN), which resembles previously reported results using other types of RFC data processing methods. Importantly, our new findings complement previously undocumented results in the following aspects: 1) the PCC and right insula are anti-correlated and tend to manifest simultaneously declines of both the negative and positive connectivity strength with subjects' age; 2) separate assessment of the negative and positive RFC metrics provides enhanced sensitivity to the aging effect; 3 ) the sensorimotor network depicts enhanced negative connectivity strength with the adult age.

Conclusion: The proposed QDA framework can produce threshold-free, voxel-wise analysis of RfMRI data the RFC metrics. The detected adult age effect is largely consistent with previously reported studies using different R-fMRI analysis approaches. Moreover, the separate assessment of the negative and positive contributions to the RFC metrics can enhance the RFC sensitivity and clarify some of the mixed results in the literature regarding to the DMN and sensorimotor network involvement in adult aging. 


\section{Highlights}

1. A quantitative data-driven analysis (QDA) framework was proposed to analysis resting-state fMRI data.

2. Threshold-free resting-state functional connectivity (RFC) metrics were derived to assess brain changes with adult age.

3. Separate assessment of the positive and negative correlations improve sensitivity of the RFC metrics.

4. The posterior cingulate and right insula cortices are anti-correlated and tend to manifest declines in both the negative and positive connectivity strength with adult age.

5. Negative connectivity strength enhances with adult age in sensorimotor network.

\section{Keywords}

Quantitative data-driven analysis (QDA), resting-state functional magnetic resonance imaging (R$f M R I)$, resting-state functional connectivity (RFC), connectivity strength index (CSI), connectivity density index (CDI), adult age.

\section{A short list of abbreviations}

QDA: Quantitative data-driven analysis

RFC: Resting-state functional connectivity

CSI: Connectivity strength index

CSIP: Positive connectivity strength index

$\mathrm{CSI}_{\mathrm{N}}$ : negative connectivity strength index

CDI: connectivity density index

$\mathrm{CDI}_{\mathrm{P}}$ : positive connectivity density index

$\mathrm{CDI}_{N}$ : negative connectivity density index 


\section{Introduction}

Among the different analysis approaches for resting-state fMRI (R-fMRI) data, the anatomic regionof-interest (ROI)-based and data-driven independent component analysis (ICA) methods are probably the most commonly used ${ }^{[1]}$. Resting-state functional connectivity (RFC) results from the ROI-based and ICA derived methods are generally similar but conceptually different. The quantitative relationship between ROI-based and ICA derived measures of RFC has been investigated with computer simulation and experiment approaches ${ }^{[2,3]}$. In theory, the ROI-based RFC measures can be shown to be the sum of the ICA derived RFC both for the within and between networks ${ }^{[2,3]}$.

With ROI-based analysis the brain is first parcellated into pre-defined anatomical regions, the mean time course for each $\mathrm{ROI}$ is then determined. By calculating the temporal correlations in a pairwise fashion between the defined ROls, for each R-fMRI dataset a correlation coefficient matrix of the ROIs can be obtained for further statistical assessment. Therefore, connectivity between specific regions is explicitly tested in a model-driven framework by using the average time course of the selected ROIs as a temporal model. Since the RFC patterns do not necessarily coincide precisely with the atlas-based ROI definition, all voxels within predefined ROls are not necessarily a part of the network-of-interest and functionally connected. This can potentially affect the accuracy and sensitivity of the ROI-based analysis ${ }^{[4]}$. On the other hand, ICA can reveal dynamics and spatially distributed brain networks in a data-driven fashion without the need of a temporal model. Despite the growing consensus regarding the ICA-derived intrinsic RFC networks in the healthy brain with stable spatial components reproduced across studies ${ }^{[5-7]}$, the precise number of independent components (NIC), as a prerequisite input parameter for ICA, is not known a priori. NIC can substantially influence the ICA outcomes ${ }^{[8]}$. Moreover, there is lack of gold standard for the selection of meaningful components to exclude non-interesting noise resources, such as ventricular, vascular, susceptibility or motion-related artifacts ${ }^{[9]}$.

In this study we refined further of our quantitative data-driven analysis (QDA) framework based on the time course of individual voxel inside the brain. The QDA approach is data-driven as ICA and can generate two types of quantitative RFC metrics for each voxel inside the brain without the need for specifying a particular threshold, model or mode. Since it uses the time course of each voxel within the brain as the seed reference in turn to compute voxel-wise whole-brain correlational coefficient matrix, the size of the correlation matrix is equal to the number of voxels inside the brain. It is typical $\mathrm{N}>10^{4}$ for whole-brain $\mathrm{R}$-fMRI datasets with $4 \mathrm{~mm}$ voxel size. To facilitate further statistical 
assessment of the whole-brain correlation matrix, we derive two types of voxel-wise RFC metrics from the correlation matrix, namely the connectivity strength index (CSI) and connectivity density index (CDI). As indicated by the names, CSI and CDI provide metrics for the local voxel connectivity strength and density with rest of the brain, respectively. These metrics can be used for straightforward statistical comparison to assess differences between groups and longitudinal changes of individuals. This is a basic requirement for radiological diagnosis in clinical practice.

It should be pointed out that there are several voxel-based RFC metrics that have been proposed in the literature to characterize the brain's resting-state activities. Among other things, the regional homogeneity ${ }^{[10-12]}$, measures of low frequency oscillation including the amplitude of low frequency fluctuations (ALFF) and the fractional amplitude of low frequency fluctuations ${ }^{[13-18]}$, measurements of complexity, such as the Hurst exponent ${ }^{[19-21]}$ and brain entropy ${ }^{[22-25]}$ have been used for studying the RFC in normal and diseased brains. These methods have yielded somewhat interesting results. However, there remains still some methodological issues to be addressed, such as the arbitrariness in the selection of cut-off frequency [13-18], loss of information [19-21], and computation difficulty [22-25]. These technical difficulties may contribute to the inconsistent findings in the published literature. Moreover, the different RFC metrics portray different aspects of R-fMRI signal and may be differently affected by the physiological activities and pathology ${ }^{[12,26]}$.

Both ICA and ROI-based approaches have previously been applied to study age-related changes in

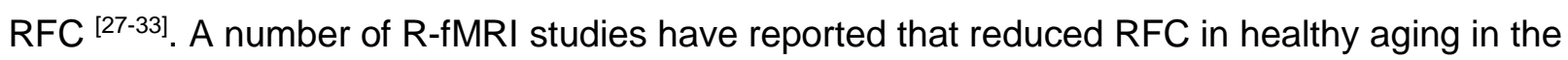
default mode network (DMN) is correlated with cognitive deficit ${ }^{29,34-37]}$. There is accumulating evidence to support the notion that elderly adults typically have reduced RFC across most parts of the DMN, particularly in the dorsal medial prefrontal cortex (MPFC) and the ventral and posterior cingulate cortex (PCC) ${ }^{[34,37]}$. However, in the reported results there is also considerable variability concerning age-related RFC differences in the limbic and other DMN subsystems. For example, some studies have found age-related RFC reduction in the hippocampal ${ }^{[34,35,37]}$ and subcortical regions ${ }^{[38]}$, whereas others reported either no significant decline or elevated RFC in some of the specific hippocampal ${ }^{[39]}$ and DMN regions ${ }^{[40,41]}$. The discrepancies in the reported findings among the different R-fMRI studies of normal aging may reflect not only variability in the sample characteristics, but also diversity in the data processing methods for deriving the different RFC metrics for connectivity of specific pathways. 
The main objective of this study is to develop a QDA framework to analyze R-fMRI data and derive intuitive and threshold-free RFC metrics, which are sensitive to physiological and pathological changes in the central nervous systems. As an application example, we used the proposed metrics to assess if and how adult age in healthy subjects influences these RFC metrics. With the proposed QDA framework we aim to provide reduced methodological complication by using a quantitative, model-free approach and more precise definitions of the RFC metrics.

\section{Experimental and Methods}

\subsection{Participants}

A total of 227 volunteers (aged 18-76 years old, male/female=99/128) completed the study and were recruited into the study through the local media advertisement in the Stockholm region. All participants were right-handed, and native Swedish speakers with normal or corrected-to-normal vision. They all reported being free of a history of neurological, psychiatric and cardiovascular diseases. None of the participants reported any use of psychotropic drugs. Each individual signed informed consent before completing the MRI examination protocol. They were financially compensated for their participation. The regional ethics committee approved the study, which was conducted in line with the declaration of Helsinki.

\subsection{MRI data acquisition protocol}

The MRI data acquisition was conducted on a whole-body 3T clinical MRI scanner (Magnetom Trio, Siemens Medical Solutions, Erlangen, Germany) equipped with a 32-channel phased-array receiving head coil. All data was acquired at Karolinska University Hospital, Huddinge, Stockholm, between noon and 5:00 PM. The MRI data acquisition protocol included the following scanning sessions: (1) 3-plane localizer; (2) Conventional clinical MRI scans including 3D T1-weighted MPRAGE, T2 and FLAIR scans; (3) A session of $375 \mathrm{~s}$ long R-fMRI measurements. The main acquisition parameters for the R-fMRI data included the following: TE/TR 35/2500 ms, flip angle $=90^{\circ}, 34$ slices of $3.5 \mathrm{~mm}$ thick, FOV $=225 \mathrm{~mm}$, matrix size $=76 \times 76$, data acquisition acceleration with GRAPPA parallel imaging method (iPAT $=2$ ), and 150 dynamic timeframes. The T1-weighted MPRAGE images used for co-registration with functional images were acquired with the following parameters: TR $=1900$ $\mathrm{ms}, \mathrm{TE}=2.52 \mathrm{~ms}, \mathrm{FA}=9$ degrees, $\mathrm{FOV}=256$, voxel size $1 \times 1 \times 1 \mathrm{~mm}$. The acquisition parameters for the FLAIR image were the following: TE/TR $=89 / 9000 \mathrm{~ms}$, flip angle $=130^{\circ}$; inversion time $(\mathrm{TI})=2500 \mathrm{~ms}$, slice thickness $=4.0 \mathrm{~mm}$, FOV=199 $\times 220 \mathrm{~mm}$. An experienced radiologist inspected both the FLAIR and T1-weighted images for potential signs of neuropathology. 


\subsection{R-fMRI data pre-processing}

The R-fMRI datasets underwent a preprocessing procedure, which was performed with AFNI (Version Debian-16.2.07 dfsg.1-3 nd14.04+1, http://afni.nimh.nih.gov/afni) and FSL (http://www.fmrib.ox.ac.uk/fsl) programs with a bash wrapper shell ${ }^{[8,42]}$. After temporal de-spiking, six-parameter rigid body image registration was performed for motion correction. The average volume for each motion-corrected time series was used to generate a brain mask to minimize the inclusion of the extra-cerebral tissues. Spatial normalization to the standard MNI template was performed using a 12-parameter affine transformation and mutual-information cost function. During the affine transformation the imaging data were also re-sampled to isotropic resolution using a Gaussian kernel with $4 \mathrm{~mm}$ full width at half maximum (FWHM). The co-registered average image volume for the cohort has 28,146 non-zero voxels inside the brain and was used to generate the average brain mask for the preprocessed whole-brain R-fMRI data with $4 \mathrm{~mm}$ spatial resolution. Nuisance signal removal was performed by voxel-wise regression using 14 regressors based on the motion correction parameters, average signal of the ventricles and their $1^{\text {st }}$ order derivatives. After baseline trend removal up to the third order polynomial, effective band-pass filtering was performed using low-pass filtering at $0.08 \mathrm{~Hz}$. Local Gaussian smoothing up to FWHM $=4 \mathrm{~mm}$ was performed using an eroded gray matter mask ${ }^{[8]}$.

Pearson's correlation coefficients (CC) were computed between the time courses of all pairs of voxels inside the brain, leading to a whole-brain functional connectivity matrix for each subject. This computation was performed for all voxels located within the brain mask, which was generated by overlapping the registered brains of all participants. This brain mask contained 28146 voxels and each voxel inside the brain was used as the seed voxel in turn. Therefore, the size of the CC matrix size is $28146 \times 28146$. Each row or column of the CC matrix corresponds to the CC image volume for the seed voxel with rest of the brain. That is the connectivity map for the seed voxel. As schematically illustrated in Fig. 1, based on the CC histogram for each row of the matrix we derived the following two types of threshold-free voxel-wise RFC metrics: the connectivity strength index (CSI) and connectivity density index (CDI). As we are interested in investigating systematically all relevant synchronized activities in the whole-brain, we quantify the negative and positive portions of the CC histogram separately to avoid information cancelation, sensitivity reduction, and statistical interference. From here on, the subscripts "N" and "P" are used to indicate the negative and positive portions of the RFC metrics, respectively. The metrics without subscripts refer to the mixed measures without distinction of the negative and position correlations. 


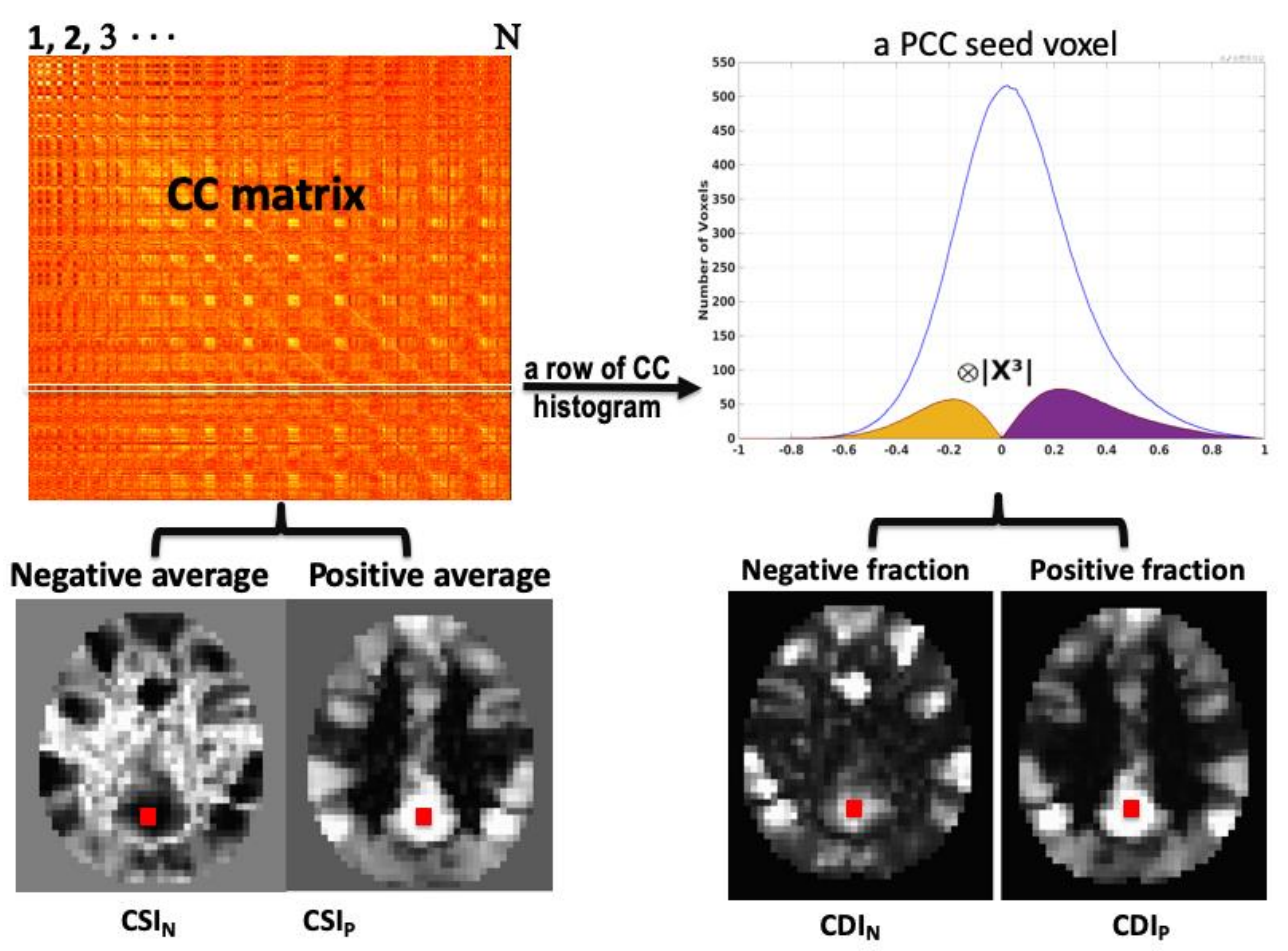

Fig. 1: A schematic overview to illustrate the QDA framework. With QDA the time course of each voxel is used in turn to compute the whole-brain CC matrix. For each row of the CC matrix, we compute a CC histogram with 200 evenly binned intervals within [-1, 1]. The histogram shown in the graph is the cohort's average CC histogram for a voxel within the PCC, whose location was marked with the red square. In QDA, two types of RFC images are derived from the CC matrix: 1) CSIP and $\mathrm{CSI}_{N}$ whose voxel values are the averages of the positives and negatives in each row of the CC matrix, respectively. 2) $C D I_{P}$ and $C D I_{N}$ whose voxel values are the positive and negative parts of the convolution between the CC histogram and the kernel, respectively.

As shown in Fig. 1, the voxel value for the $\mathrm{CSI}_{\mathrm{P}}$ and $\mathrm{CSI}_{\mathrm{N}}$ are defined as the averages of the positives and negatives in each row of the CC matrix, respectively. That is

$$
\begin{aligned}
& C S I_{\mathrm{P}}=\left(\sum_{C C>0} C C_{\text {row }}\right) / n p \\
& C S I_{N}=\left(\sum_{C C<0} C C_{\text {row }}\right) / n n
\end{aligned}
$$

Where $C C_{\text {row }}$ refers to a row in the CC matrix. $n p$ and $n n$ refer to the number of positive and negative correlation coefficients in a row of the CC matrix, respectively. The voxel values for CDI are defined as the convolution between the $\mathrm{CC}$ histogram and a kernel function. That is

$$
\mathrm{CDI}=\mathrm{Hist}\left(C C_{\text {row }}\right) \otimes \mathrm{kernel}
$$


Similar to the $\mathrm{CSI}_{\mathrm{P}}$ and $\mathrm{CSI}_{\mathrm{N}}$, the $\mathrm{CDI}_{\mathrm{P}}$ and $\mathrm{CDI}_{\mathrm{N}}$ correspond to the positive and negative portions of the convolution defined in eq. [3], respectively. To facilitate statistical comparison, it is useful to transform the raw RFC metrics into standard Z-score using the following formula:

$$
Z=(\mathrm{RFC}-\mathrm{u}) / \sigma
$$

Where $\mu$ and $\sigma$ are the mean and standard dilation of the corresponding RFC metrics, respectively. Fig. 2 shows an axial slice of the average $\mathrm{CSI}_{\mathrm{N}}$ and $\mathrm{CSI}_{\mathrm{P}}$ for the cohort before and after the Z-score transform. For optimization of the CDI sensitivity, we investigated 6 different kernel functions, including

$$
\begin{aligned}
& \mathrm{k}_{\mathrm{i}=1,2, \ldots 4}=\left|\mathrm{x}^{\mathrm{i}}\right|, \\
& \mathrm{k}_{5}=\left|\sin ^{2}(\pi / 2 \mathrm{x})\right|, \\
& \mathrm{k}_{6}=\operatorname{step}(|\mathrm{x}|-0.3),
\end{aligned}
$$

where $x \subset[-1,1]$ corresponding to the interval of the correlation coefficients. The kernels are also graphically depicted in Fig. 3. It is obvously that a kernel weights the higher correlation coefficients more than the lower ones. The widely used threshold approach can be considered as the case of the square-well kernel function $k_{6}$. For illustration, an arbitrary threshold of 0.3 was used here. The CSI metrics can also be considered as a special case of CDI corresponding to a kernel of the sign function.
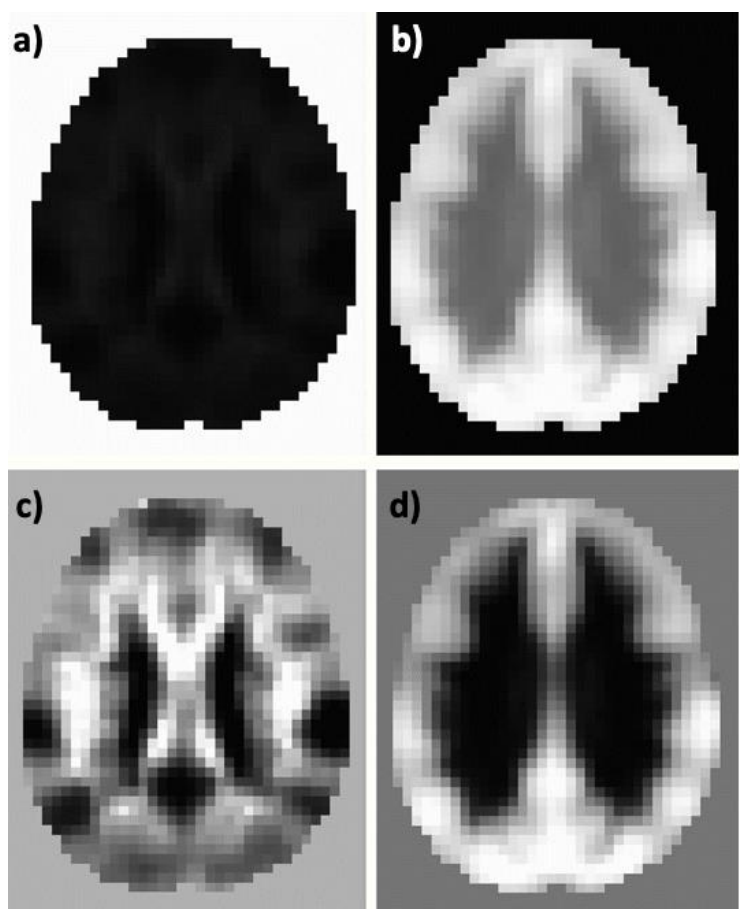

Fig. 2: An axial slice of the average $\operatorname{CSI}_{N}(a)$ and $\mathrm{CSI}_{\mathrm{P}}(\mathrm{b})$ for the cohort before $(\mathrm{a}, \mathrm{b})$ and after the Zscore transformation $(c, d)$. 


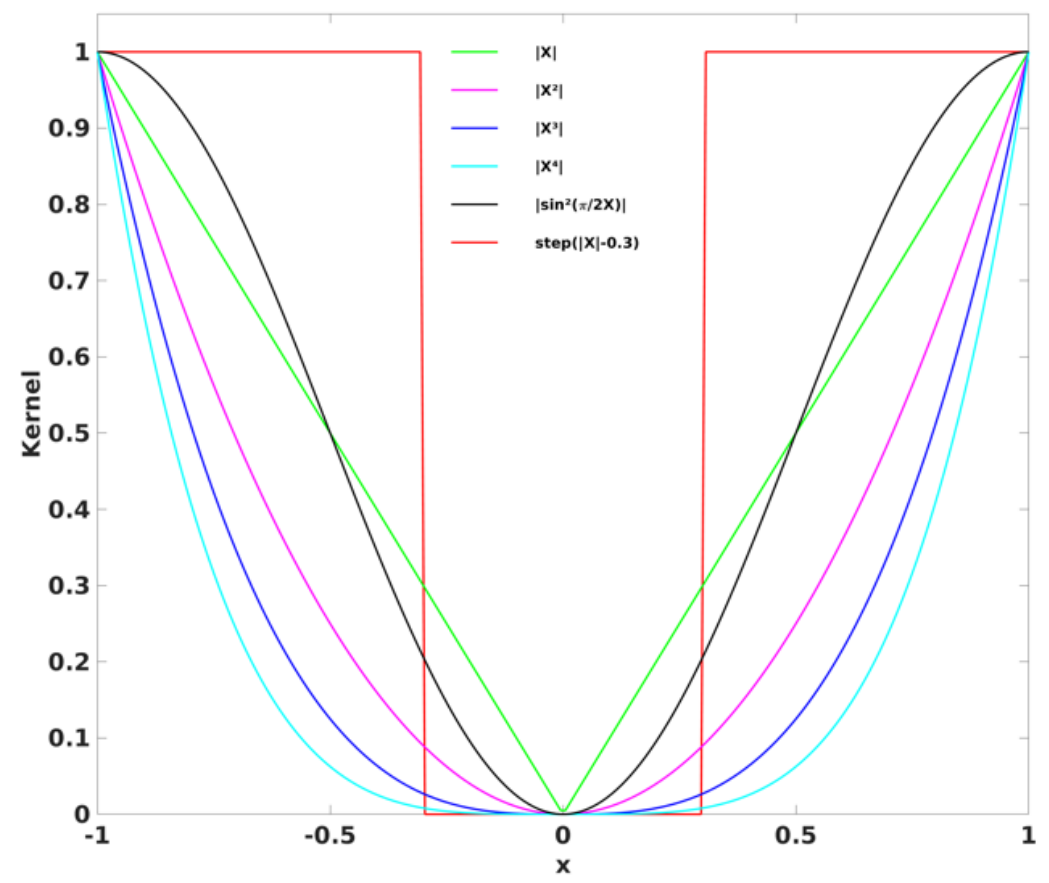

Fig. 3: The six different kernel functions investigated in the study to derive the $\mathrm{CDI}_{\mathrm{P}}$ and $\mathrm{CDI}_{N}$ metrics. The widely used threshold method can be considered as the case for the square-well kernel function $\left(\mathrm{k}_{6}\right)$.

\subsection{Statistical analyses}

To investigate if and how the RFC metrics are influenced by the adult age for the studied cohort we performed voxel-wise linear regression analyses of the CSI and CDI metrics versus the subject's age, while the gender was treated a covariate by using the AFNI program 3dRegAna to extract the regression parameter $\beta$ and linear coefficient $r$. The statistical significance was assessed by using a two-step approach. Firstly, we imposed a voxel-wise threshold $p<0.001$ (uncorrected corresponding t-score $>=3.34$ ) to form the initial cluster candidates. Secondly, we performed permutation simulations without assuming a particular form of probability distribution for the voxel values in the statistic images to identify the brain regions of interest (ROI) out of the initially detected clusters at family-wise error rate (FWER) $p \leq 0.05$. Using the detected ROls as masks, we evaluated the mean values of the RFC metrics for each ROI and made scattered plot against the subjects' age. Besides linear regression analysis with age, we performed also verification using student t-test between the young and elderly subgroups. For this, we selected all subjects aged 18-30 years old as the young subgroup ( $n=124$, males/females=51/73), and all subjects aged 64-76 years old as the elderly subgroup ( $n=76$, males/females=35/41). To keep sufficient age gap between the young and elderly subgroups the remaining 27 subjects in the age range of 31-63 years old were excluded from the ttests. 


\section{Results}

\subsection{The QDA framework}

As expected, the characteristics of the CC histogram for each seed voxel in the brain is dependent on its location in the brain. Fig. 4 shows the average CC histogram of the cohort for a seed voxel in the PCC as illustrated by the green cross and red square in Fig. 1. The histogram is somewhat asymmetric and shifted toward the positive side. This is quite typical at least for voxels within gray matter. Selecting different threshold values along the histogram allows us to observe the RFC networks of different connection strengths associated with the selected PCC seed voxel. As shown in Fig. 4, at high negative CC threshold (Figs. 4a and b) we observe the DMN. At low negative and positive CC thresholds we observe its association with cerebral spinal fluid (CSF) space and white matter (Figs. 4c and d). At moderately high positive CC threshold, the PCC is not only a part of the DMN, but also connected to most of the cortical gray matter (Fig. 4e). At high positive CC threshold the PCC is associated with the posterior portion of the DMN and the visual cortex (Fig. 4f). For further illustration, we selected 4 seed voxels located in different brain regions (see Fig. 5 and Table 1) and tissue types for further investigation.
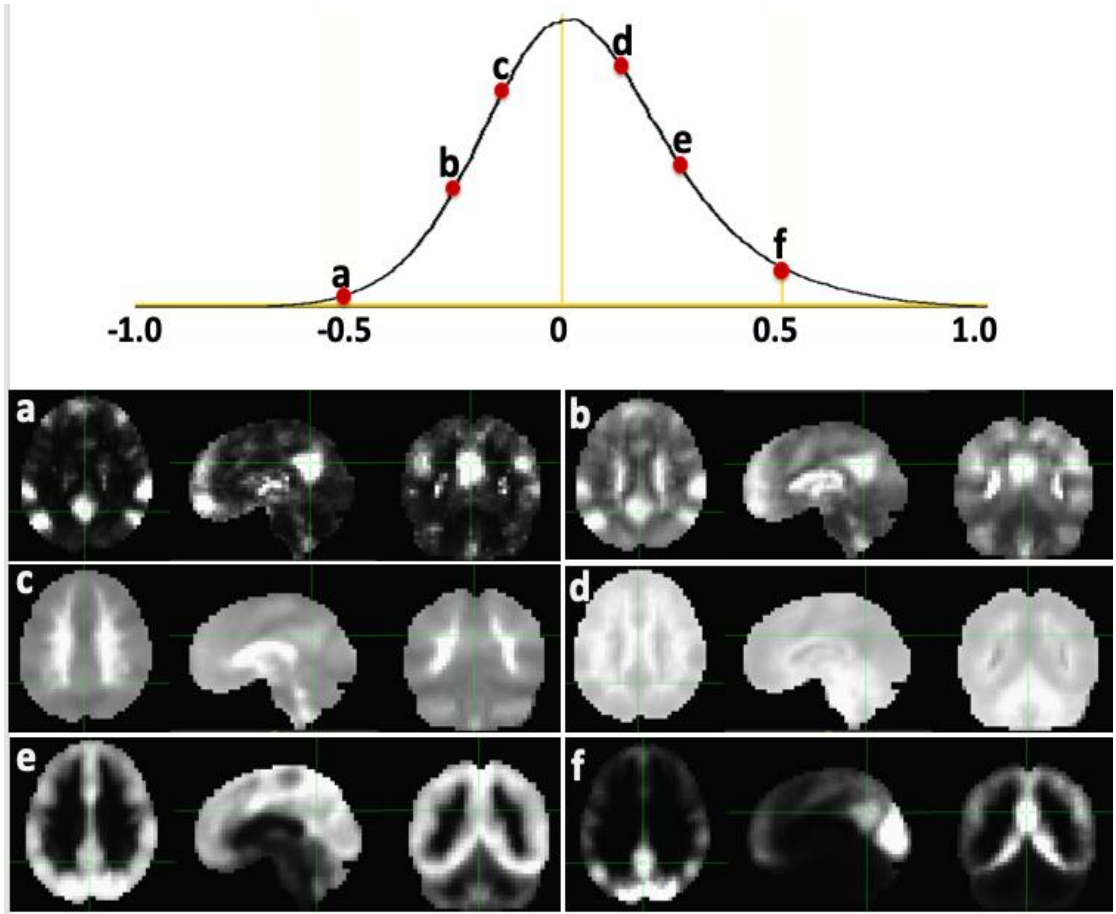

Fig. 4: The average CC histogram of the cohort for a seed voxel in the posterior cingulate cortex (PCC) as indicated by the green cross. Selecting different threshold values along the histogram allows us to detect the functional connection networks of different strengths (a-f) associated with the seed voxel in the PCC. 

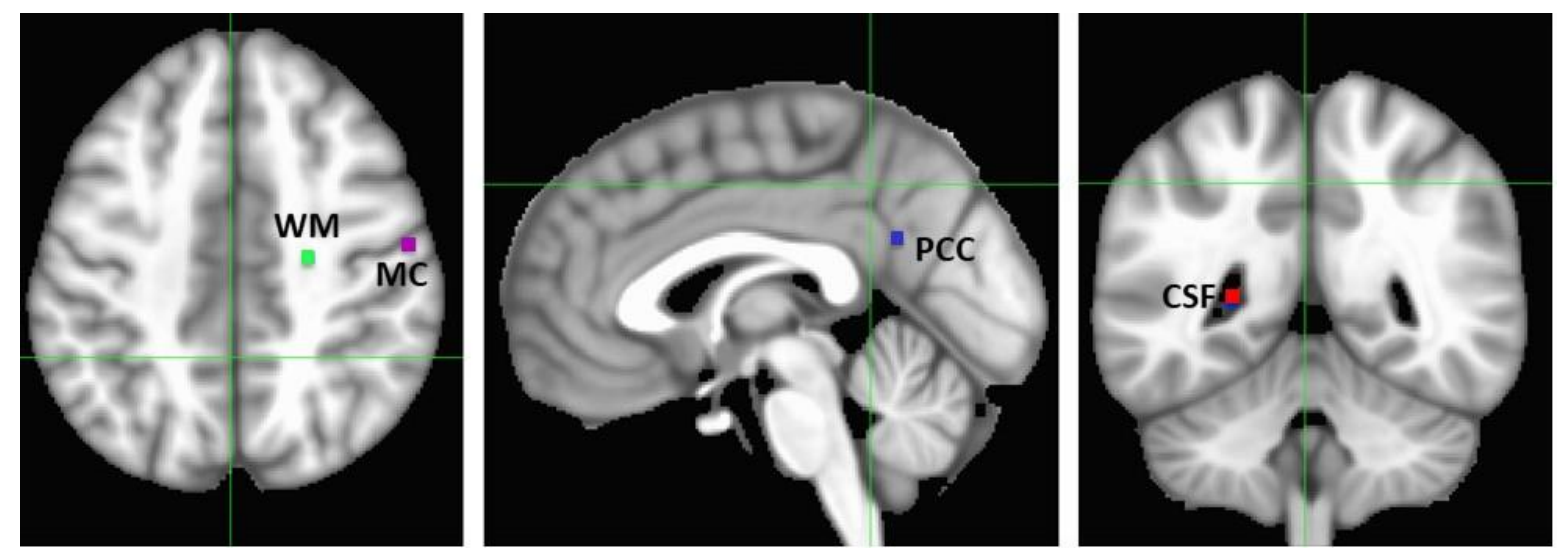

Fig. 5: Anatomic locations for 4 different seed voxels of different tissue types including white matter (WM), cerebral spinal fluid (CSF), PCC and motor cortex (MC).

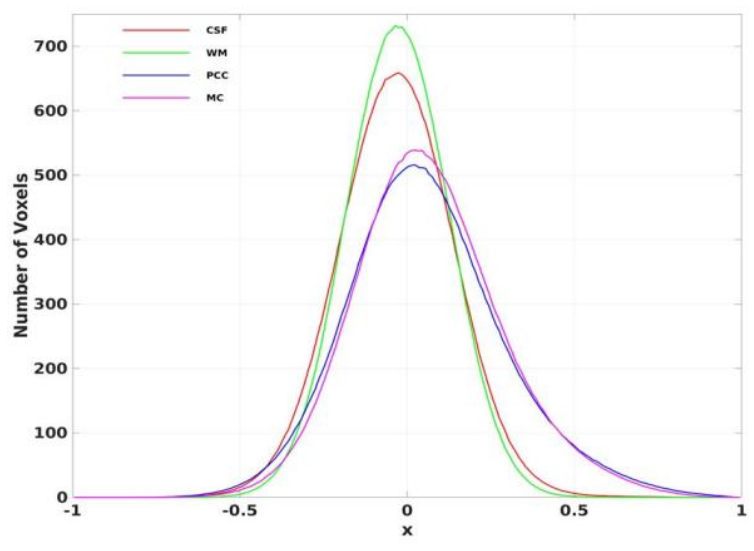

Fig. 6: The average CC histograms of the cohort for the 4 different seed voxels shown in Fig. 5.

As shown in Fig. 6, the histogram for the seed voxel in the MC is quite similar to that for the PCC with a long positive tail, whereas the histograms for the seed voxels in white matter and CSF regions are overall narrower and the peak is slightly shifted toward the negative side. The convolutions of thse CC histograms are depicted in Fig. 7. For the white matter and CSF seeds, the negative portions are more dominant, while the positive parts of the convolutions are larger for the grey matter seed voxels in the PCC and MC. For the polynomial kernels, increasing the order of the polynomial shifts the peak values of the convolutions away from " 0 ". Selecting different kernels can, therefore, adjust the contrast and sensitivity of the derived $C D I_{P}$ and $C D I_{N}$ metrics. Fig. 8 shows an axial slice of $\mathrm{CDI}_{\mathrm{P}}$ and $\mathrm{CDI} \mathrm{I}_{\mathrm{N}}$ images for a typical R-fMRI dataset acquired from a 36 years old male subject. A number of brain regions depict disproportionally high CDIp including the bilateral medial prefrontal cortex (mPFC), superior and middle temporal gyri (MTG), inferior and superior parietal lobule (SPL), precuneus and PCC. As suggested in previously published studies, these regions have been described as RFC hubs so as to imply their important role in neural signaling and communication 
across the brain ${ }^{[43,44]}$. On the other hand, the PCC, insula cortex. White matter and CSF regions have usually high $\mathrm{CDI}_{N}$ metric. The contrast and intensity variations across the rows in Fig. 8 demonstrate that the kernel function can influence the contrast and signal-to-noise ratio (SNR) of the CDI metrics.
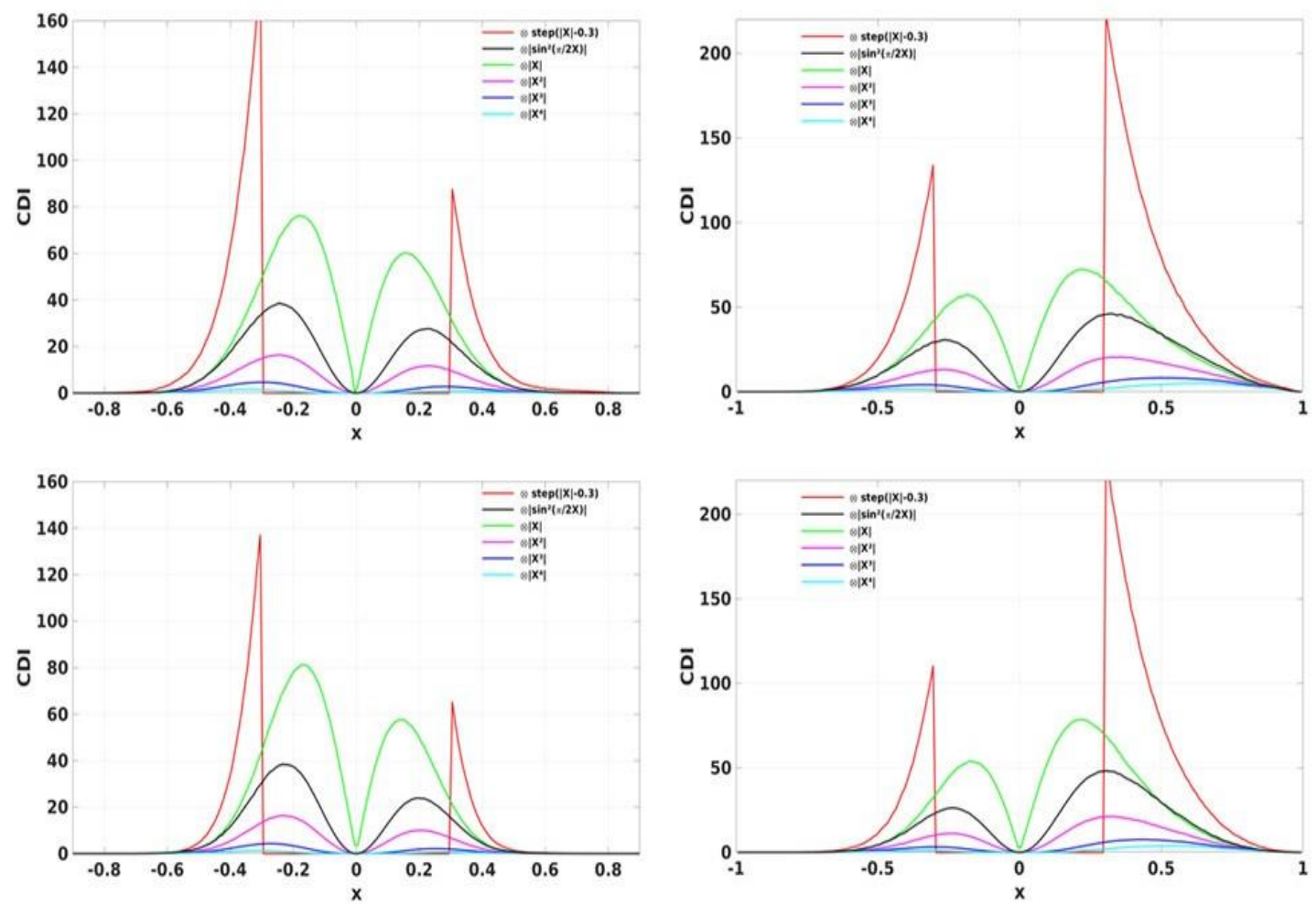

Fig. 7: The convolutions of the CC histograms shown in Fig. 6 for the 4 different seed voxels located in WM (a), PCC (b), CSF(c) and MC (d).

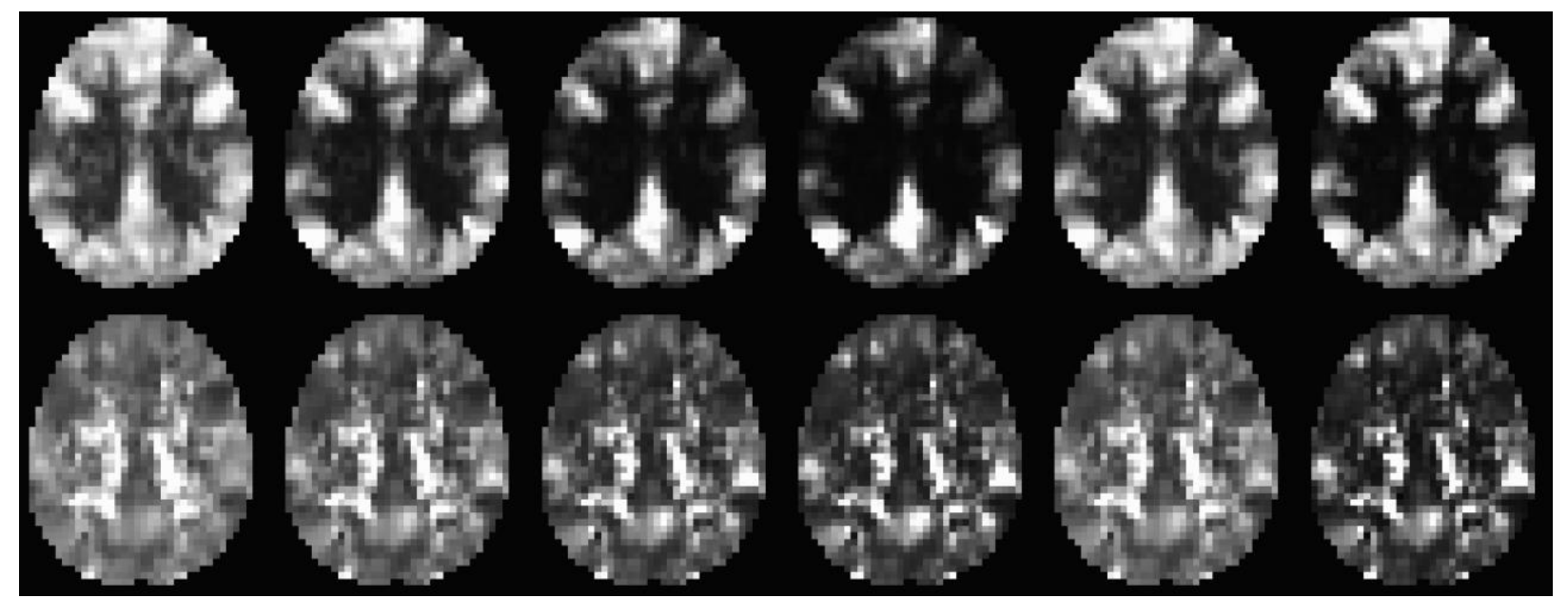

Fig. 8: An axial slice of the $\mathrm{CDI}_{\mathrm{P}}$ (upper row) and $\mathrm{CDI}_{\mathrm{N}}$ (lower row) metrics derived from a typical RfMRI dataset (a male subject of 36 years' age). The images from left to right depict the results for the following 6 kernel functions $|x|,\left|x^{2}\right|,\left|x^{3}\right|,\left|x^{4}\right|, \sin ^{2}(\pi / 2 x)$ and $\operatorname{step}(|x|-0.3)$, respectively. 

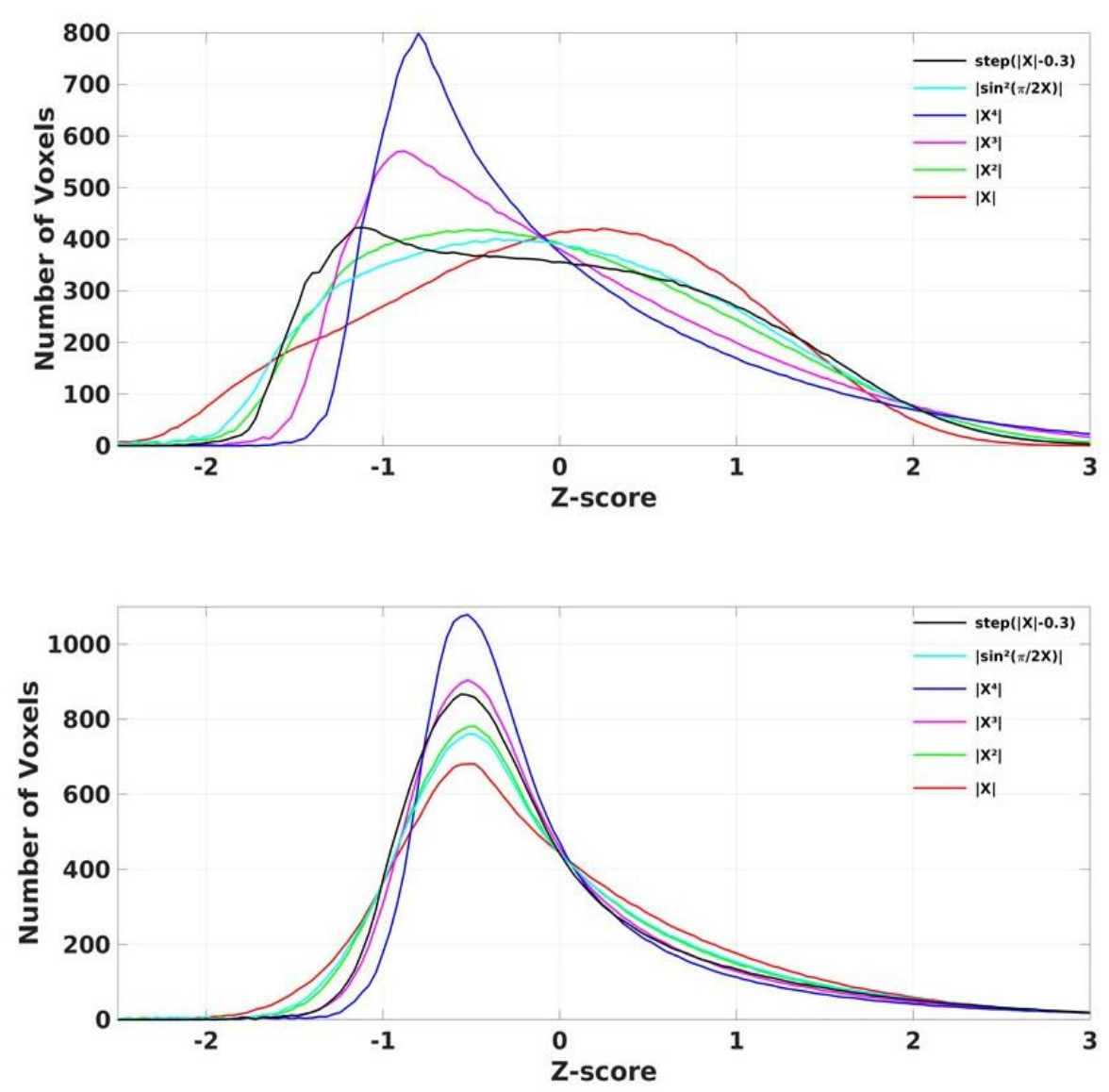

Fig. 9: The average $\operatorname{CDI}_{\mathrm{P}}(\mathrm{a})$ and $\mathrm{CDI}_{\mathrm{N}}(\mathrm{b})$ histograms for the cohort derived by using the 6 different kernels $k_{1}-k_{6}$.

A more quantitative comparison of the kernel effect can be better appreciated in the average CDI $_{P}$ and $\mathrm{CDI}_{N}$ histograms for the cohort shown in Fig. 9. It is clear that the kernel functions can influence the distributions of the RFC metrics and therefore the statistics based on it.

\subsection{Overall trends of the RFC metrics with the adult age}

To compare the overall trend of advanced age effect on the RFC, we analyzed the RFC histograms for the young and elderly subgroups. Fig. 10 depicts the histogram differences between the young and elderly subgroups for the CDIP and CDI $\mathrm{C}_{\mathrm{P}}$ metrics. Positive peaks above the "0" horizontal line indicate that the young subgroup has more voxel populations than the elderly subgroup for the zscore ranges corresponding to the peaks, whereas the negative peaks indicate the opposite. As expected, the kernel functions affect the number of peaks, the shapes and positions. Fig. 11 shows the total area of the peaks both above and below the horizontal line as a function of the kernels. The $\mathrm{CDI}_{\mathrm{P}}$ histogram differences between the young and elderly subgroups are systematically larger than 
those for the $\mathrm{CDI}_{N}$ metrics, indicating CDI metrics may have higher sensitivity to the aging effect. Especially for the polynomial based kernels $\left(\mathrm{k}_{1}-\mathrm{k}_{4}\right)$, increasing the order of the polynomials improves the sensitivity for the CDIP metrics and also the contrast between the $\mathrm{CDI}_{\mathrm{P}}$ and $\mathrm{CDI}_{\mathrm{N}}$ metrics, as demonstrated by the increased gaps between the $\mathrm{CDI}_{\mathrm{P}}$ and $\mathrm{CDI}_{N}$ results shown in Fig. 11.

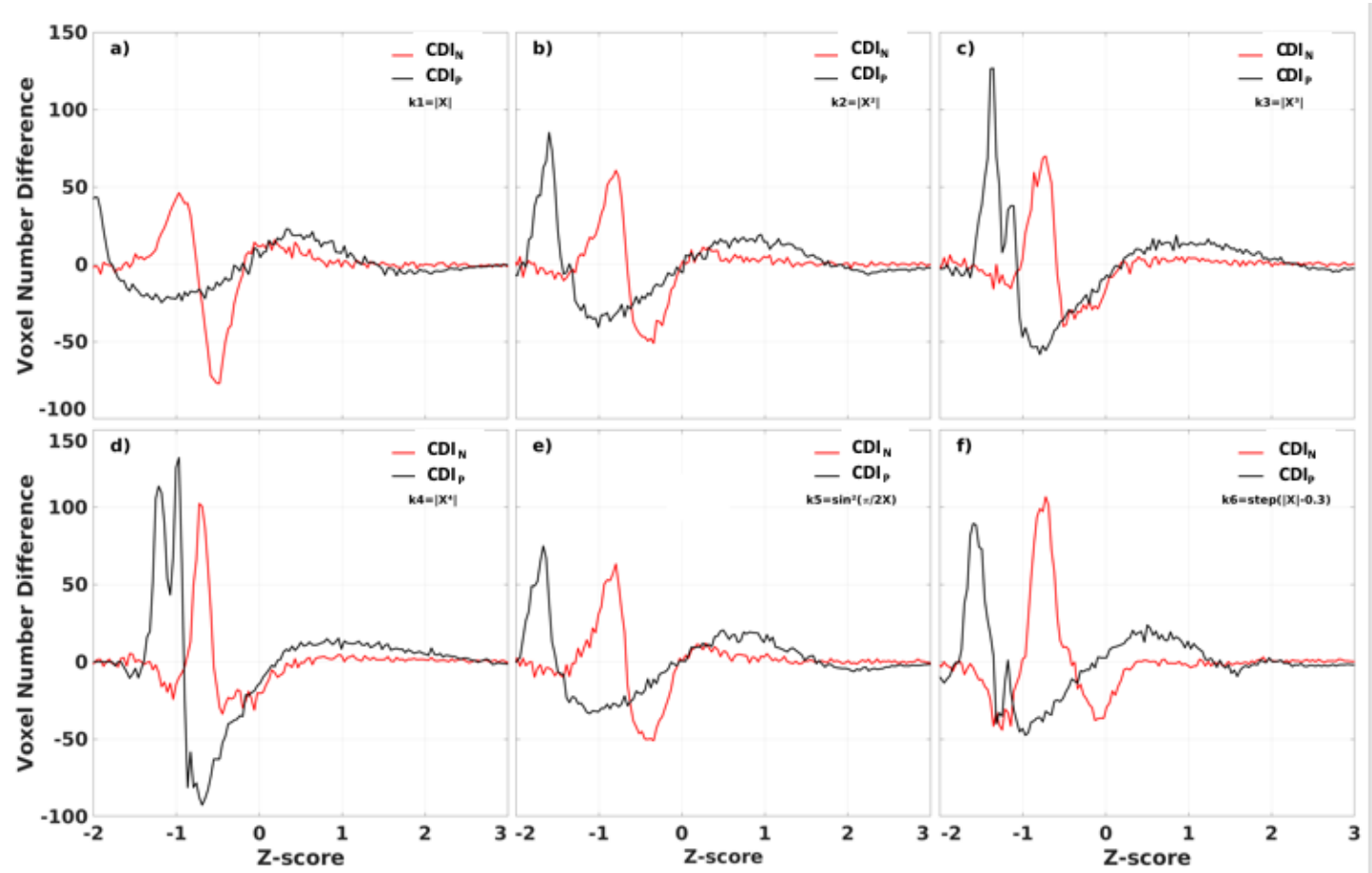

Fig. 10: The differences in CDI histograms between the young and elderly sub-groups. The CDIN and $\mathrm{CDI}_{\mathrm{p}}$ results for the 6 different kernels are shown.

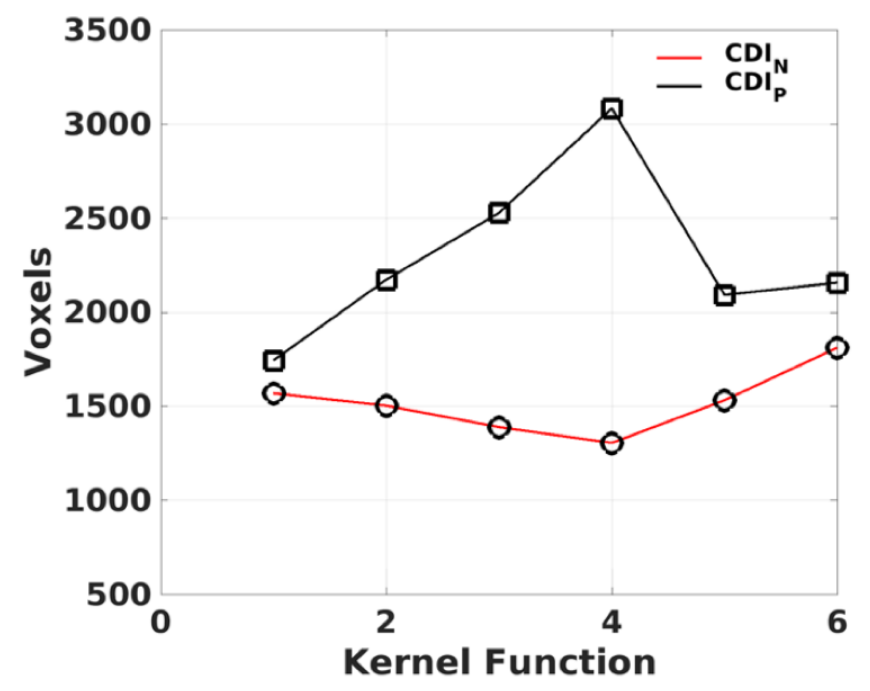

Fig. 11: The $\mathrm{CDI}_{\mathrm{P}}$ and $C D \mathrm{I}_{\mathrm{N}}$ histogram differences between the young and elderly sub-groups as a function of the kernels. Each data point corresponds to the total area above and below the ' 0 ' horizontal line of the histogram differences shown in Fig. 10. 


\subsection{RFC changes associated with the adult age}

Fig. 12 and Table 2 shows the linear regression results for the CSI, CSIN and CSIP data versus subjects' age. The CSI metric without separation of the negative and positive correlations shows decline of the functional connectivity strength with age in the superior and middle prefrontal gyrus (MFG) and increase of connectivity strength in the precuneus and right inferior parietal lobule ( $r$-IPL). The more specifically defined $\mathrm{CSI}_{N}$ and $\mathrm{CSI}_{\mathrm{P}}$ metrics are more sensitive to the adult age effect and the detected brain volumes with significant aging effect are nearly tripled compared with that for the CSI metric. With $\mathrm{CSI}_{\mathrm{N}}$ and CSIP we observe also a more intricate pattern of change with the adult age, which are summarized as follows:

(1) The CSIP shows mainly decline trend with adult age (negative $\beta$ and $r$ ) in the extended DMN including superior and MFG, PCC, bilateral insula cortex and left middle temporal gyrus (I-MTG) except for putamen where up-regulation of CSIp was observed.

(2) The $\mathrm{CSI}_{N}$ depicts a more complicated pattern of dependence on the adult age. The negative connectivity strength was reduced (positive $\beta$ and $r$ ) with the adult age in the PCC, right insula cortex and IPL, while enhancement (negative $\beta$ and $r$ ) was detected in the sensorimotor network (paracentral lobule, bilateral postcentral gyri), bilateral para hippocampal cortices (PHC), and right superior temporal gyrus ( $r-S T G)$.

(3) There are two brain regions where both the $\mathrm{CSI}_{\mathrm{N}}$ and $\mathrm{CSI} \mathrm{P}_{\mathrm{P}}$ demonstrated significant reduction trend with the adult age, which were detected by applying the logical "AND" operation to the regression results for the $\mathrm{CSI}_{\mathrm{P}}$ and $\mathrm{CSI}_{\mathrm{N}}$. As shown in Table 2 and Fig. 13, the two overlapping ROIs in the PCC and r-insula cortex depict significant down-regulation of CSIP and $\mathrm{CSI}_{\mathrm{N}}$ metrics with the subjects' age.

Using the above overlapping ROls as the seed masks, we computed the Pearson's correlation maps for the time courses of the seeds, which are displayed in in Fig. 13. As expected, the associated RFC network for the ROI in the PCC is obviously the well-known DMN and include 4 negatively correlated brain regions, which are the bilateral IPL and insula cortices. On the other hand, the associated RFC network for the ROI in the right insular cortex includes the PCC and bilateral precuneus as the negatively correlated brain regions. Fig 14. Shows the anti-correlated brain regions between the above 2 RFC networks obtained by applying a multiplication of the above two correlation maps associated with the 2 overlapping ROls and thresholding at $\mathrm{CC} \leq(-0.5)$. It is clear that the mutually inclusive anti-correlation between the PCC and the right insular cortex are likely the reason why both $\mathrm{CSI}_{\mathrm{P}}$ and $\mathrm{CSI}_{\mathrm{N}}$ metrics in these regions depict declines with the adult age. 


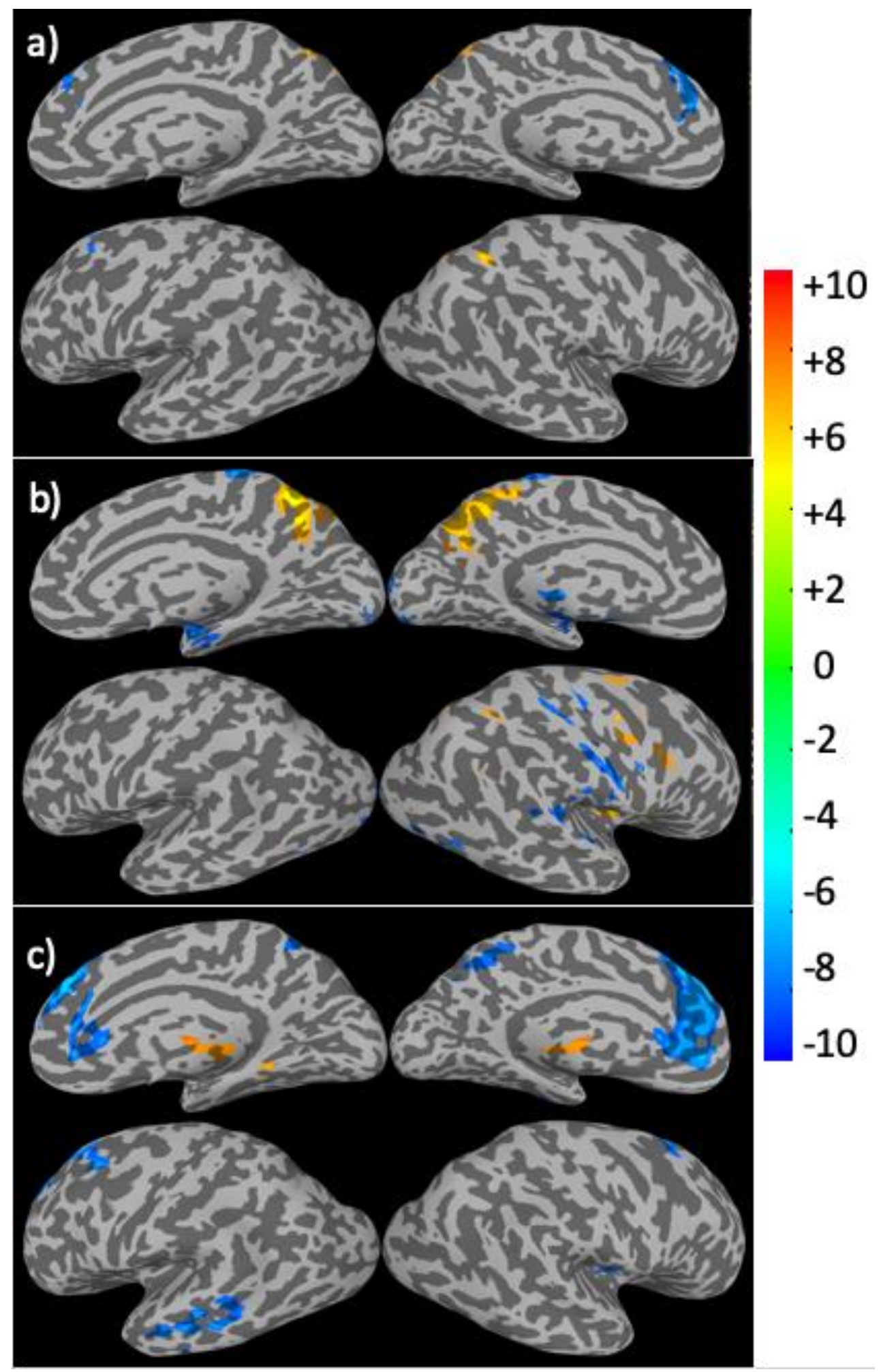

Fig. 12: Brain regions with significant correlation $(p<0.05$, corrected) between the connectivity strength metrics and the subject's age. The results for the CSI (a), CSI $(b)$ and $C S I_{P}(c)$ are depicted separately. The Color bar shows the t-score level. 

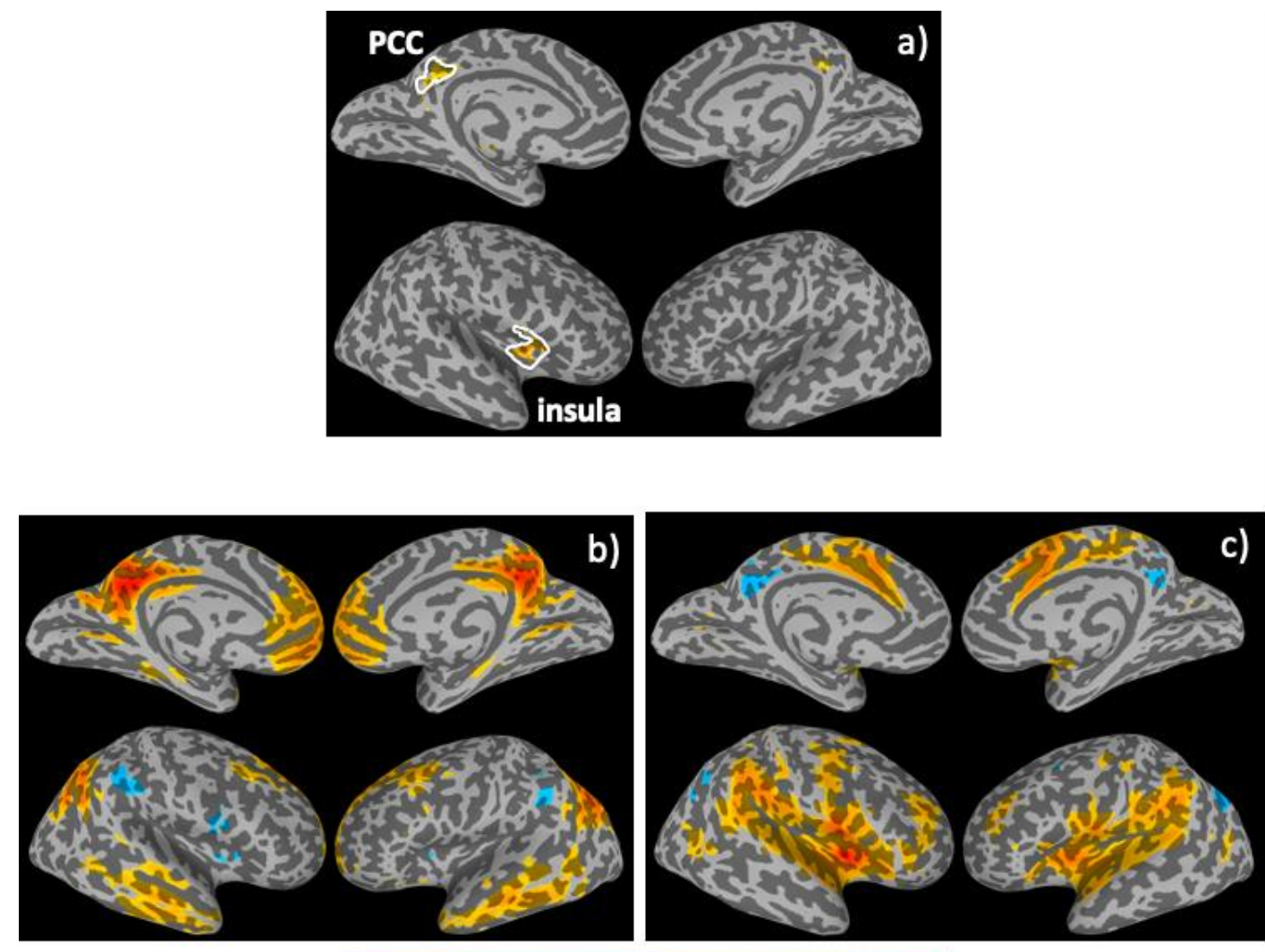

$\begin{array}{llllllllllll}-10 & -8 & -6 & -4 & -2 & 0 & 2 & 4 & 6 & 8 & 10\end{array}$

Fig. 13: The overlapping ROIs in the PCC and right insula cortex where both the $\mathrm{CSI}_{\mathrm{P}}$ and $\mathrm{CSI}_{\mathrm{N}}$ metrics depict significant decline with the adult age (a). The average Pearson's correlation maps of the cohort associated the overlapping ROI seeds in the PCC (b) and insula cortex (c).
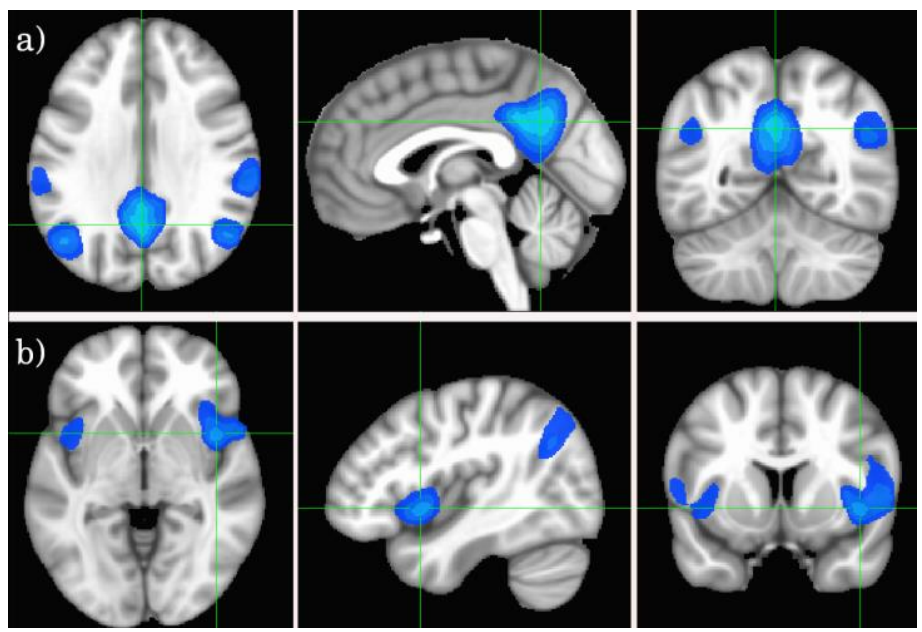

Fig. 14: Cross-sectional display of the anti-correlation networks associated with the 2 overlapping ROIs as derived from the product of the maps shown in Figs. $13 b$ and $13 c$ at $C C \leq(-0.5)$. The crossing points of green lines depict the center of mass for each ROI. 
a)

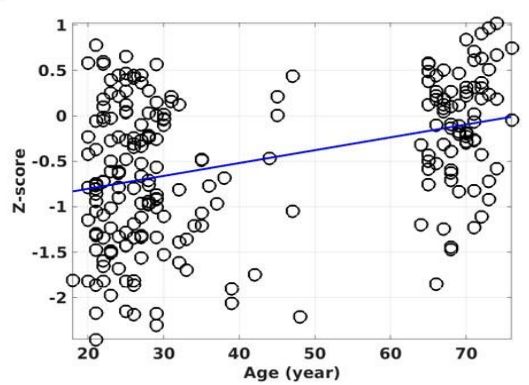

c)

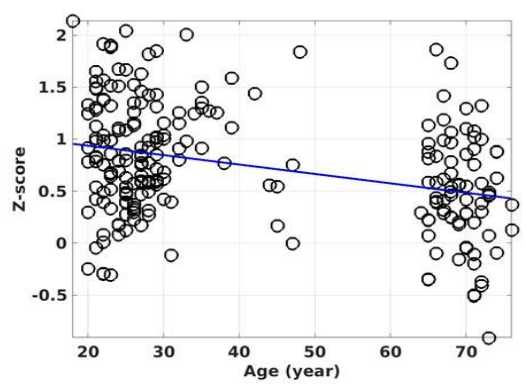

b)
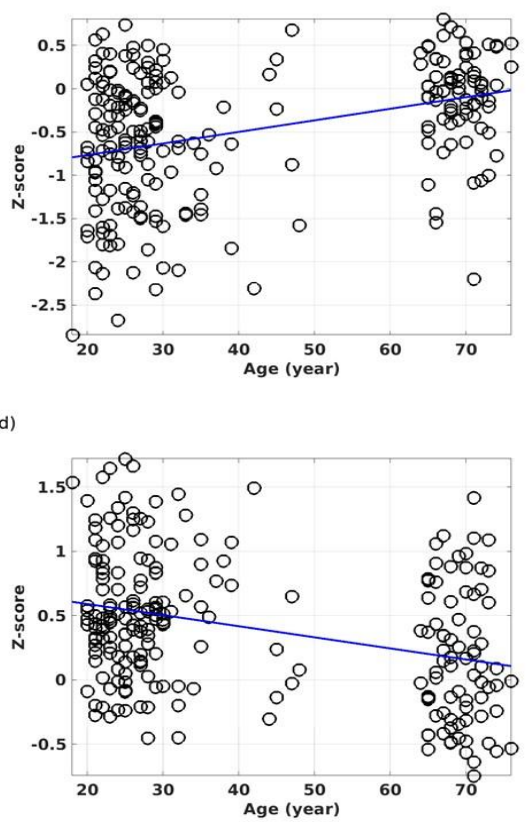

Fig. 15: The ROI average of the CSI $\mathrm{N}_{\mathrm{N}}$ metric against the subject's age for the overlapping ROI in the PCC (a). The ROI average for the CSI $\mathrm{N}_{N}$ metric against the subject's age for the overlapping ROI in the right insula cortex (b). The ROI average for the CSIP metric against the subject's age for the overlapping ROI in the PCC (c). The ROI average for the CSIP metric against the subject's age for the overlapping $\mathrm{ROI}$ in the insula cortex (d). The lines show the linear regression results of the RFC metrics against the subject's ages.

Fig. 15 shows the ROI average of the $\mathrm{CSI}_{N}$ and $\mathrm{CSI}_{\mathrm{P}}$ metrics in the PCC and right insula cortex as a function of the subject's age. With normal aging, both the $\mathrm{CSI}_{P}$ and $\mathrm{CSI}_{N}$ are reduced in these overlapping brain regions. Therefore, the PCC and right insula are particularly sensitive to the adult age effect. However, the aging effect is barely detectable by the unseparated CSI metric (see Table 2). Fig. 16 shows the detected brain volumes where the $\mathrm{CDI}_{\mathrm{P}}$ and $\mathrm{CDI}_{N}$ metrics are significantly associated with the adult age. As expected, the $\mathrm{CDI}_{\mathrm{P}}$ and $\mathrm{CDI}_{\mathrm{N}}$ metrics derived by using the different kernels differ in their sensitivity in detecting the adult age effect. The trend shown in Fig. 16a is quite similar to that of the histogram result shown in Fig. 11b, although the effect shown in Fig. 11b are overall larger (nearly doubled). This is because the histogram results in Fig. 11b did not impose any statistical criterion, while the linear regression results shown in Fig. 16a are subjected to statistical criterion for significance and accidental noise contributions are excluded. The sensitivity difference of the kernels is also manifested in the regression parameter $\beta$ which are detailed in Table 3 and Fig $16 b$. 

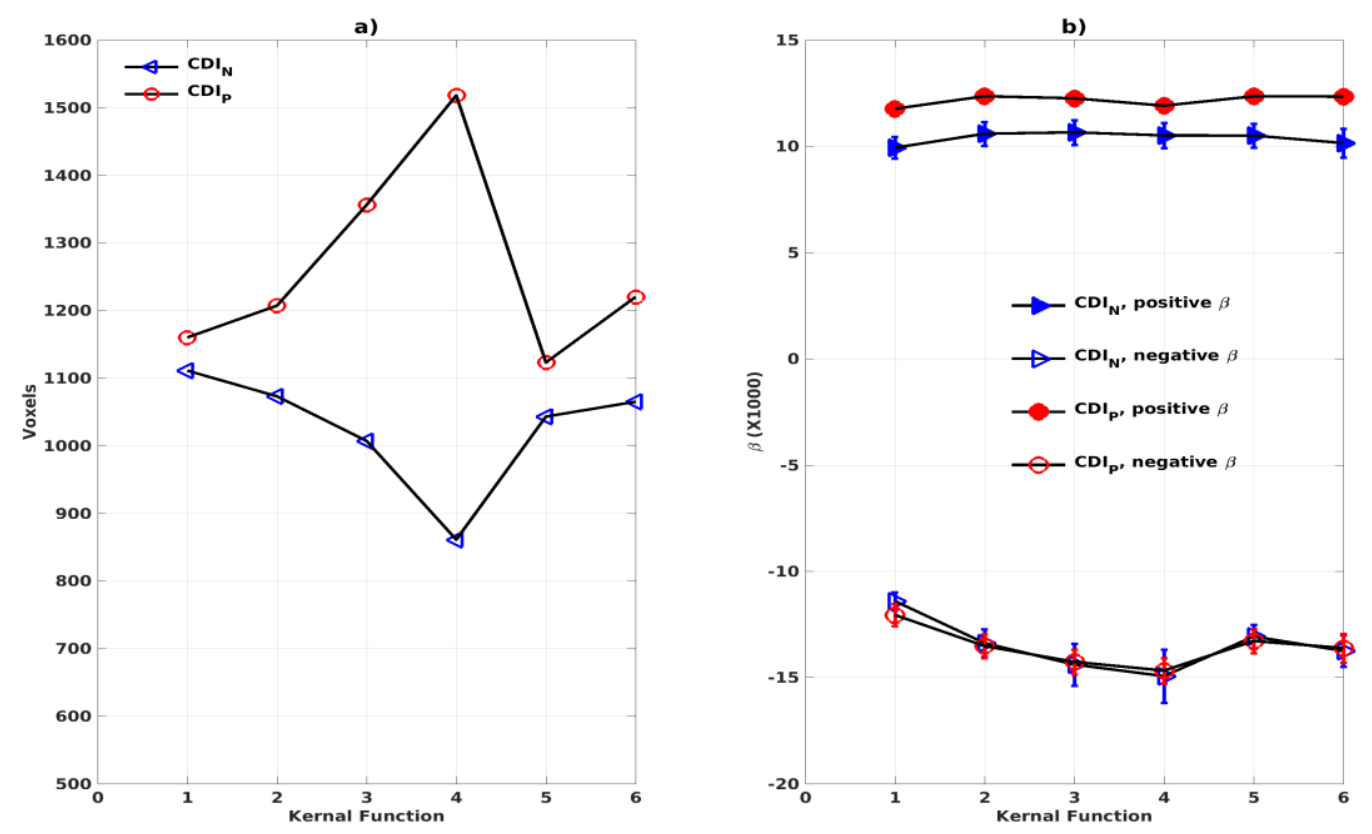

Fig. 16: The total volumes of the detected brain regions with significant correlation $(p<0.05$, corrected) between the connectivity density index $\left(C D I_{P}\right.$ and $\left.C D I_{N}\right)$ and the subject's age as a function of the kernels (a). The average regression parameter $\beta$ for the detected brain regions as a function of the kernels (b). The negative and positive correlations were assessed separately.

To compare the similarity of the detected aging effects among the CDI metrics of different kernels, we assessed the joint overlapping brain regions detected by the different $\mathrm{CDI}_{\mathrm{N}}$ metrics of different kernels. This was also performed for the CDIp results of different kernels. The observed overall trends of RFC enhancement or decline with age are quite similar. The joint overlapping volumes for the $C D I_{P}$ and $C D I_{N}$ metrics of different kernels are 733 and 671 voxels, respectively. Moreover, there is also a reasonable anatomic consistency between the results of the connectivity strength metrics and connectivity density metrics. As detailed in Tables 2 and 3 , the anatomical locations of the joint overlapping regions for the different CDIP metrics match those for the 3 largest ROls identified by the CSIP results (see Table 2). Similarly, the brain regions of the joint overlapping for the different CDIN metrics are largely the same as those identified by the CSIN data (see Table 2). However, it should be noted that the $\beta$ parameters for the $C D I_{N}$ and $C S I_{N}$ have opposite signs even through the trend of change with the adult age is the same. This is because the negative connectivity strength $\left(C S I_{N}\right)$ is negative in nature, while the connectivity density corresponding to the negative correlation $\left(C D I_{N}\right)$ is always positive. Therefore, the enhancement of the negative connectivity strength $\left(C S I_{N}\right)$ with age (for example in the sensorimotor network) corresponds to a negative $\beta$, while the connectivity density result corresponds to a positive $\beta$ value. 


\section{Discussion}

\subsection{Effects of adult age on RFC}

Age is an important risk factor for declines of neural cognitive functions and pathology of neurodegenerative diseases. It is also a complex metric that is difficult to interpret precisely the involved physiology. Healthy individuals of similar age may have quite different vascular and brainhealth status. It follows that age is not a single strongest predictor for the RFC in the brain. This is likely to be the reason why the linear regressions of the RFC metrics with the adult age depict substantial scatters and relative low correlation coefficients. The impact of the potential confounds and pre-processing strategies that can mitigate them have been extensively investigated in the published literature ${ }^{[27-33,45,46]}$. Here we focus on comparing our findings in the context of documented literature results, particularly the adult age effect in the DMN, dorsal attention network (DAN), sensorimotor network and subcortical brain regions.

With QDA, we found support for RFC decline with advancing adult age in multiple brain regions of the DMN and DAN, including superior and MFG, PCC, MTG, and IPL. Age-related RFC decrements in the DMN and DAN have previously been reported in numerous R-fMRI studies using ROI and ICA based analysis $[27,35,37,47,48]$. Our findings regarding to the RFC changes in the DMN are overall in agreement with previous reported results ${ }^{[35,38,40,41,49-53]}$. Besides the DWMN and DAN, normal aging was associated with RFC increase in the sensorimotor, subcortical network, and parahippocampal cortex. This has also been reported previously [40, 45, 46, 48,51,54]. We didn't find significant age-related RFC declines in precuneus and specific sub-regions of the hippocampal cortex as reported in previous studies ${ }^{[40,41]}$. Since we assessed the negative and positive correlation separately, this may allow us to detect more intricate age-related RFC changes in the brain. To illustrate this point, we analyzed further the 3 ROls with significant correlation between the CSI and the subject's age. As shown in Tables 2 and 4 and Fig. 17, the detected ROI in the precuneus depicted significant positive linear correlation between CSI and the subject's age $\left(\beta=9.50 \times 10^{-3}\right.$, $r=0.459$ ), even though the CSIP and $\mathrm{CSI}_{N}$ in the same ROI showed only a slight (not significant) increment and decrement with age, respectively. i.e., contribution from a non-significant CSIP increment and a non-significant $\mathrm{CSI}_{N}$ decrement resulted in a significant increment trend in the CSI metric. With the same line of reasoning, we can explain why the MFG ROI detected by the CSI metric is much smaller than that detected by the CSIP metric, because the decremental trend in the CSIP metric was partially canceled by the CSIN contribution. This can also explain why we didn't detect significant CSI decrement with the adult age in the PCC and R-insula, because both the CSIP and $\mathrm{CSI}_{N}$ metrics exhibited significant decremental trends with age and their contributions annulled 
each other. Therefore, it is important to pay attention to the precise definition of the RFC when comparing the results of different studies.
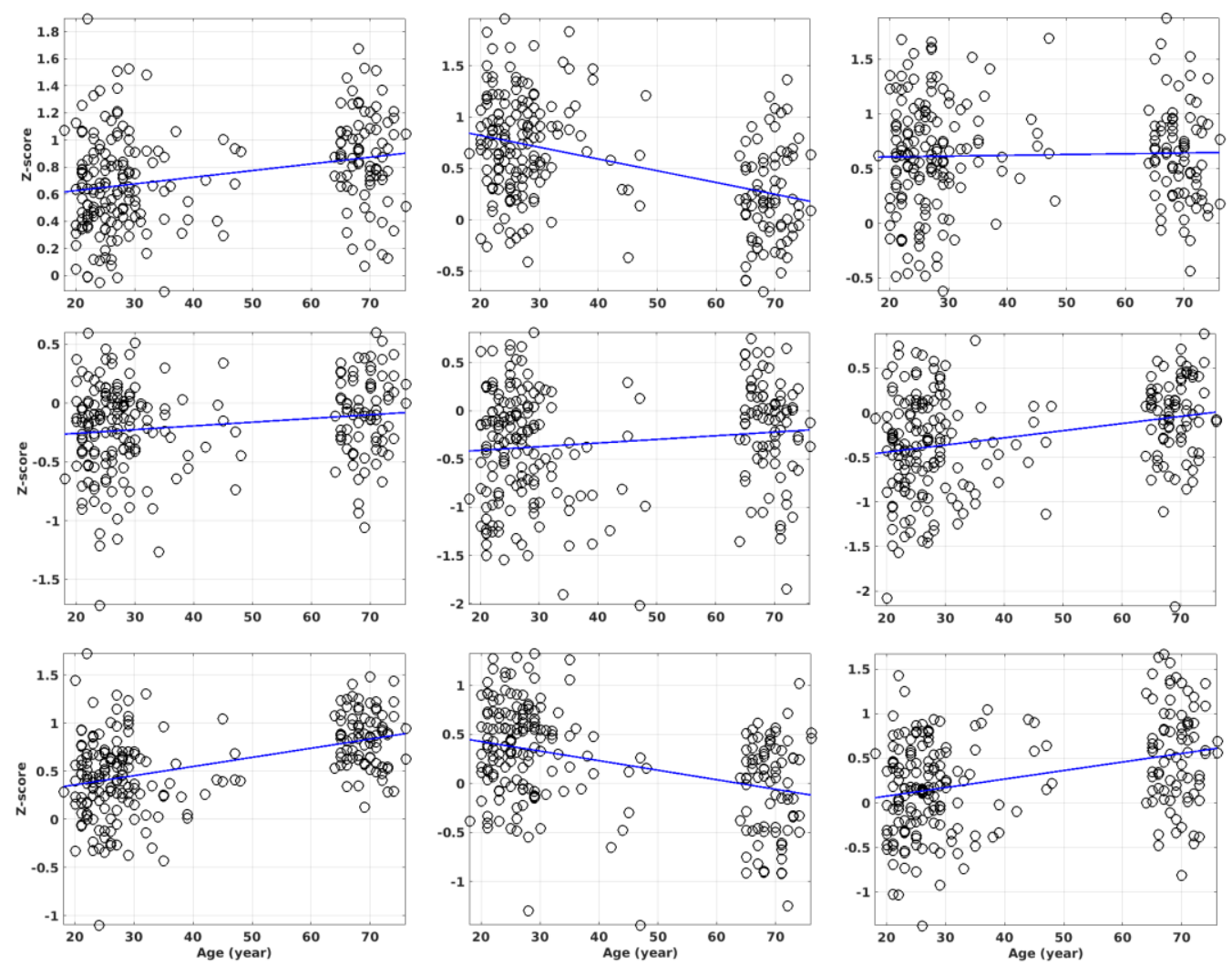

Fig. 17: The ROI average of the CSIP, CSIN and CSI metrics against the subject's age for the 3 ROIs with significant correlation between CSI and the subject's age. The columns 1 to 3 are the results for the ROIs in the precuneus, MFG, and R-IPL, respectively. The rows 1 to 3 are the results for the $\mathrm{CSI}_{\mathrm{P}}, \mathrm{CSI}_{\mathrm{N}}$ and CSI metrics, respectively. The ROI masks are solely based on the CSI metric only.

\subsection{Methodological issues}

The QDA framework proposed in the study is a voxel-wise and data-driven approach. It has the following two unique features: 1) It can avoid confounding caused by the cancellation of the negative and positive correlations by assessing the negative and positive portions of the CC histogram separately; 2) It derives different RFC metrics based on the connectivity strength and density by utilizing the concept of convolutions with different kernels. The metrics weight all the correlations of a given voxel with the rest of the brain according to the amplitudes of the correlation coefficients and disregard the anatomical distance between the correlation pairs. This permits a comprehensive characterization of the intrinsic activities of each voxel without the use of an arbitrary threshold. The QDA approach can encapsulate the widely used threshold approach as a special case of the squarewell kernel function. Even the CSI metrics can be encapsulate under the convolution concept for a 
special kernel of the sign function. This provides a unified view for RFC and can facilitate its further optimization.

The current results based on the QDA framework should be interpreted in the context of some technical and biological limitations. Firstly, at a TR of $2500 \mathrm{~ms}$, the cardiac and respiratory fluctuation effects might be aliased into the low frequency R-fMR signal fluctuations. The regression up-to the 1 st order derivative of the head motions and lowpass filtering could not completely eliminate the effects of these physiological noises ${ }^{[55-58]}$. Thus, these aliasing effects could reduce the specificity of the RFC metrics, or even might further confound the detected RFC differences between the young and elderly sub-groups. With the more up-to-date acquisition techniques, such as multi-band simultaneous acquisition of multiple slices and compress-sensing with high under-sampling factor, it is possible to use a shorter TR (e.g., $500 \mathrm{~ms}$ ) and higher spatial resolution for the data acquisition. Therefore, these physiological effects may be further mitigated.

Secondly, the resting state is associated with spontaneous thoughts and cognitive processing, we cannot exclude the possibility that differences in spontaneous thoughts may exist between the young and elderly subjects ${ }^{[59]}$. However, considering the overall consistency of our results with the previous studies, particularly the results form the longitudinal studies ${ }^{[60-63]}$, it is unlikely that these differences have major influence on our findings. These initial findings encourage the future use of QDA as a tool to analyze longitudinal R-fMRI data aimed to develop a comprehensive understanding of age- or pathology-related brain functional changes.

Thirdly, the generalizability, or external validity issue should be considered. This is due to the non-random recruitment procedures and relying on a sample of convenience. The sample size used in this study $(\mathrm{N}=227)$ is moderate, includes unbalanced young and elderly subgroups reflecting the difficulties to recruit elderly healthy subjects. The ages of the participants range from young to old adulthood (reflecting the age of participants in most neuroimaging studies). The age-related RFC differences observed in this study were relatively small but quite robust. However, the results from this cross-sectional study of the cohort cannot distinguish whether the RFC changes in the brain regions are due to gradual changes throughout the adulthood or a more sudden change at later stage in life.

\subsection{Negative cross correlation, white matter and CSF}

As discussed above negative correlation is an important fraction of the CC histogram irrespective of the tissue type and anatomical location of the voxel in question. In published literature, there is also a rapid growing interest in studying the negative correlations between the voxels ${ }^{[64-72]}$. It is clear that 
the negative portion of the CC histogram is more dominant for voxels in CSF ${ }^{[73]}$ and white matter ${ }^{\text {[74- }}$ 77]. However, the negative portion cannot be ignored even for voxels in the grey matter. To avoid confound caused by inappropriate preprocessing pipelines, we have carefully tested and updated our preprocessing pipeline. We did not implement the global signal regression (GSR) which removes the mean signal averaged over the entire brain. GSR removal via linear regression is one of the most

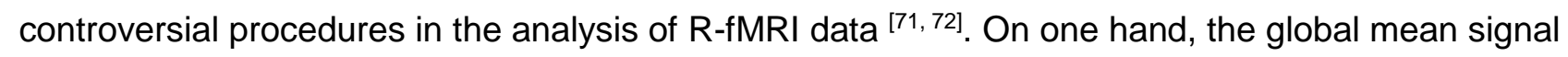
contains variance associated with respiratory, scanner-, and motion-related artifacts. Its removal by GSR can improve various quality control metrics, which enhances the anatomical specificity of RFC networks, and increase the explained behavioral variance. On the other hand, GSR alters the distribution of regional signal correlations in the brain, can induce artefactual anti-correlation patterns, may remove real neural signal, and can distort RFC metrics. The brain masked 'global signal' is usually misunderstood, because it is not 'global' and its variance contains dominant contributions from different domains of the voxels with temporally coherent signal variation.

To limit the study in a reasonable scope, in the discussion of the adult age effect on RFC we focused on grey matter and did not discuss white matter and CSF related issues. However, it should be pointed out that aging effects in white matter ${ }^{[74-77]}$ and CSF ${ }^{[73,78]}$ are also worth exploring. There is indeed a rapid growing interest in these arenas in published literature ${ }^{[73-78]}$, particularly in the context of the age effect for the glymphatic system.

\section{Conclusions}

The proposed QDA framework can data-drive, provide threshold-free and voxel-wise analysis of RfMRI data and offer a unified view for RFC metrics which can facilitate further development and optimization of the RFC metrics by choosing appropriate kernel functions. The QDA results for the adult age effect are largely consistent with previously published results based on other analysis methods. Moreover, our new findings based on the separate assessment of the negative and positive correlations can improve the sensitivity of the RFC metrics to physiological changes associated with the advancing adult age and may clarify some of the confounding reports in the literature regarding to the $\mathrm{DMN}$ and sensorimotor network involvement in normal aging.

\section{Author contributions}

Xia Li: Conceptualization and software. Håkan Fischer: Project administration, editing, supervision and funding acquisition. Amirhossein Manzouri: Validation and visualization. Kristoffer N.T Månsson: Investigation and data curation. Tie-Qiang Li: Methodology, formal analysis and writing original draft preparation. 


\section{Acknowledgements}

We would acknowledge the experimental assistance and stimulating discussion with the colleagues at the ${ }^{4}$ Department of Medical Radiation and Nuclear Medicine, Karolinska University Hospital. This work was supported by China Scholarship Council, Zhejiang Natural Science Foundation of China (No. LY18E070005), Key Research and Development Program of Zhejiang Province (No. 2020C03020), and Stockholm Regional ALF fund.

\section{References}

1. J. M. Tyszka, D. P. Kennedy, L. K. Paul, R. Adolphs, Largely typical patterns of resting-state functional connectivity in high-functioning adults with autism. Cereb Cortex 24, 1894-1905 (2014).

2. C. Rosazza, L. Minati, F. Ghielmetti, M. L. Mandelli, M. G. Bruzzone, Functional connectivity during restingstate functional MR imaging: study of the correspondence between independent component analysis and region-of-interest-based methods. AJNR Am J Neuroradiol 33, 180-187 (2012).

3. S. E. Joel, B. S. Caffo, P. C. van Zijl, J. J. Pekar, On the relationship between seed-based and ICA-based measures of functional connectivity. Magn Reson Med 66, 644-657 (2011).

4. X. Song, L. P. Panych, N. K. Chen, Data-Driven and Predefined ROI-Based Quantification of Long-Term Resting-State fMRI Reproducibility. Brain Connect 6, 136-151 (2016).

5. E. A. Allen, E. B. Erhardt, E. Damaraju, W. Gruner, J. M. Segall, R. F. Silva, M. Havlicek, S. Rachakonda, J. Fries, R. Kalyanam, A. M. Michael, A. Caprihan, J. A. Turner, T. Eichele, S. Adelsheim, A. D. Bryan, J. Bustillo, V. P. Clark, S. W. Feldstein Ewing, F. Filbey, C. C. Ford, K. Hutchison, R. E. Jung, K. A. Kiehl, P. Kodituwakku, Y. M. Komesu, A. R. Mayer, G. D. Pearlson, J. P. Phillips, J. R. Sadek, M. Stevens, U. Teuscher, R. J. Thoma, V. D. Calhoun, A baseline for the multivariate comparison of resting-state networks. Front Syst Neurosci 5, 2 (2011).

6. S. M. Smith, P. T. Fox, K. L. Miller, D. C. Glahn, P. M. Fox, C. E. Mackay, N. Filippini, K. E. Watkins, R. Toro, A. R. Laird, C. F. Beckmann, Correspondence of the brain's functional architecture during activation and rest. Proc Natl Acad Sci U S A 106, 13040-13045 (2009).

7. J. Damoiseaux, S. Rombouts, F. Barkhof, P. Scheltens, C. Stam, S. Smith, C. Beckmann, Consistent restingstate networks across healthy subjects. Proceedings of the National Academy of Sciences 103, 13848 (2006).

8. Y. Wang, T. Q. Li, Dimensionality of ICA in resting-state fMRI investigated by feature optimized classification of independent components with SVM. Frontiers in human neuroscience 9, 259 (2015).

9. Y. Wang, T. Q. Li, Analysis of whole-brain resting-state FMRI data using hierarchical clustering approach. PLoS One 8, e76315 (2013).

10. Y. Zang, T. Jiang, Y. Lu, Y. He, L. Tian, Regional homogeneity approach to fMRI data analysis. Neuroimage 22, 394-400 (2004).

11. T. B. Meier, P. S. F. Bellgowan, A. R. Mayer, Longitudinal assessment of local and global functional connectivity following sports-related concussion. Brain Imaging Behav 11, 129-140 (2017).

12. B. B. Reynolds, A. N. Stanton, S. Soldozy, H. P. Goodkin, M. Wintermark, T. J. Druzgal, Investigating the effects of subconcussion on functional connectivity using mass-univariate and multivariate approaches. Brain Imaging Behav, (2017).

13. Y. Sun, Z. Dai, Y. Li, C. Sheng, H. Li, X. Wang, X. Chen, Y. He, Y. Han, Subjective Cognitive Decline: Mapping Functional and Structural Brain Changes-A Combined Resting-State Functional and Structural MR Imaging Study. Radiology 281, 185-192 (2016). 
14. X. D. Zhang, X. L. Jiang, Z. Cheng, Y. Zhou, Q. Xu, Z. Q. Zhang, R. Qi, S. Luo, Y. S. Yun, H. J. Chen, X. Kong, G. M. Lu, L. J. Zhang, Decreased Coupling Between Functional Connectivity Density and Amplitude of Low Frequency Fluctuation in Non-Neuropsychiatric Systemic Lupus Erythematosus: a Resting-Stage Functional MRI Study. Mol Neurobiol, (2016).

15. P. Pan, L. Zhu, T. Yu, H. Shi, B. Zhang, R. Qin, X. Zhu, L. Qian, H. Zhao, H. Zhou, Y. Xu, Aberrant spontaneous low-frequency brain activity in amnestic mild cognitive impairment: A meta-analysis of resting-state fMRI studies. Ageing Res Rev 35, 12-21 (2017).

16. H. Yang, X. Y. Long, Y. Yang, H. Yan, C. Z. Zhu, X. P. Zhou, Y. F. Zang, Q. Y. Gong, Amplitude of low frequency fluctuation within visual areas revealed by resting-state functional MRI. Neuroimage 36, 144152 (2007).

17. Y. F. Zang, Y. He, C. Z. Zhu, Q. J. Cao, M. Q. Sui, M. Liang, L. X. Tian, T. Z. Jiang, Y. F. Wang, Altered baseline brain activity in children with ADHD revealed by resting-state functional MRI. Brain Dev 29, 8391 (2007).

18. Q. H. Zou, C. Z. Zhu, Y. Yang, X. N. Zuo, X. Y. Long, Q. J. Cao, Y. F. Wang, Y. F. Zang, An improved approach to detection of amplitude of low-frequency fluctuation (ALFF) for resting-state fMRI: fractional ALFF. $J$ Neurosci Methods 172, 137-141 (2008).

19. S. Hayasaka, P. J. Laurienti, Comparison of characteristics between region-and voxel-based network analyses in resting-state fMRI data. Neuroimage 50, 499-508 (2010).

20. B. J. He, Scale-free properties of the functional magnetic resonance imaging signal during rest and task. J Neurosci 31, 13786-13795 (2011).

21. P. Ciuciu, P. Abry, B. J. He, Interplay between functional connectivity and scale-free dynamics in intrinsic fMRI networks. Neuroimage 95, 248-263 (2014).

22. D. B. de Araujo, W. Tedeschi, A. C. Santos, J. Elias, Jr., U. P. Neves, O. Baffa, Shannon entropy applied to the analysis of event-related fMRI time series. Neuroimage 20, 311-317 (2003).

23. M. Zhao, C. Zhou, Y. Chen, B. Hu, B. H. Wang, Complexity versus modularity and heterogeneity in oscillatory networks: combining segregation and integration in neural systems. Phys Rev E Stat Nonlin Soft Matter Phys 82, 046225 (2010).

24. Y. Jia, H. Gu, Q. Luo, Sample entropy reveals an age-related reduction in the complexity of dynamic brain. Sci Rep 7, 7990 (2017).

25. A. Viol, F. Palhano-Fontes, H. Onias, D. B. de Araujo, G. M. Viswanathan, Shannon entropy of brain functional complex networks under the influence of the psychedelic Ayahuasca. Sci Rep 7, 7388 (2017).

26. A. M. Golestani, J. B. Kwinta, Y. B. Khatamian, J. J. Chen, The Effect of Low-Frequency Physiological Correction on the Reproducibility and Specificity of Resting-State fMRI Metrics: Functional Connectivity, ALFF, and ReHo. Front Neurosci 11, 546 (2017).

27. E. L. Dennis, P. M. Thompson, Functional brain connectivity using $\mathrm{fMRI}$ in aging and Alzheimer's disease. Neuropsychol Rev 24, 49-62 (2014).

28. G. Alarcon, A. Cservenka, M. D. Rudolph, D. A. Fair, B. J. Nagel, Developmental sex differences in resting state functional connectivity of amygdala sub-regions. Neuroimage 115, 235-244 (2015).

29. B. B. Biswal, M. Mennes, X. N. Zuo, S. Gohel, C. Kelly, S. M. Smith, C. F. Beckmann, J. S. Adelstein, R. L. Buckner, S. Colcombe, A. M. Dogonowski, M. Ernst, D. Fair, M. Hampson, M. J. Hoptman, J. S. Hyde, V. J. Kiviniemi, R. Kotter, S. J. Li, C. P. Lin, M. J. Lowe, C. Mackay, D. J. Madden, K. H. Madsen, D. S. Margulies, H. S. Mayberg, K. McMahon, C. S. Monk, S. H. Mostofsky, B. J. Nagel, J. J. Pekar, S. J. Peltier, S. E. Petersen, V. Riedl, S. A. Rombouts, B. Rypma, B. L. Schlaggar, S. Schmidt, R. D. Seidler, G. J. Siegle, C. Sorg, G. J. Teng, J. Veijola, A. Villringer, M. Walter, L. Wang, X. C. Weng, S. Whitfield-Gabrieli, P. Williamson, C. Windischberger, Y. F. Zang, H. Y. Zhang, F. X. Castellanos, M. P. Milham, Toward discovery science of human brain function. Proc Natl Acad Sci U S A 107, 4734-4739 (2010).

30. R. L. Bluhm, E. A. Osuch, R. A. Lanius, K. Boksman, R. W. Neufeld, J. Theberge, P. Williamson, Default mode network connectivity: effects of age, sex, and analytic approach. Neuroreport 19, 887-891 (2008). 
31. I. Weissman-Fogel, M. Moayedi, K. S. Taylor, G. Pope, K. D. Davis, Cognitive and default-mode resting state networks: do male and female brains "rest" differently? Hum Brain Mapp 31, 1713-1726 (2010).

32. C. Zhang, N. D. Cahill, M. R. Arbabshirani, T. White, S. A. Baum, A. M. Michael, Sex and Age Effects of Functional Connectivity in Early Adulthood. Brain Connect 6, 700-713 (2016).

33. X. N. Zuo, C. Kelly, A. Di Martino, M. Mennes, D. S. Margulies, S. Bangaru, R. Grzadzinski, A. C. Evans, Y. F. Zang, F. X. Castellanos, M. P. Milham, Growing together and growing apart: regional and sex differences in the lifespan developmental trajectories of functional homotopy. J Neurosci 30, 15034-15043 (2010).

34. K. L. Campbell, O. Grigg, C. Saverino, N. Churchill, C. L. Grady, Age differences in the intrinsic functional connectivity of default network subsystems. Front Aging Neurosci 5, 73 (2013).

35. J. S. Damoiseaux, C. F. Beckmann, E. J. Arigita, F. Barkhof, P. Scheltens, C. J. Stam, S. M. Smith, S. A. Rombouts, Reduced resting-state brain activity in the "default network" in normal aging. Cereb Cortex 18, 1856-1864 (2008).

36. L. K. Ferreira, G. F. Busatto, Resting-state functional connectivity in normal brain aging. Neurosci Biobehav Rev 37, 384-400 (2013).

37. D. Scheinost, E. S. Finn, F. Tokoglu, X. Shen, X. Papademetris, M. Hampson, R. T. Constable, Sex differences in normal age trajectories of functional brain networks. Hum Brain Mapp 36, 1524-1535 (2015).

38. M. Ystad, T. Eichele, A. J. Lundervold, A. Lundervold, Subcortical functional connectivity and verbal episodic memory in healthy elderly--a resting state fMRI study. Neuroimage 52, 379-388 (2010).

39. L. Pasquini, M. Scherr, M. Tahmasian, C. Meng, N. E. Myers, M. Ortner, M. Muhlau, A. Kurz, H. Forstl, C. Zimmer, T. Grimmer, A. M. Wohlschlager, V. Riedl, C. Sorg, Link between hippocampus' raised local and eased global intrinsic connectivity in AD. Alzheimers Dement 11, 475-484 (2015).

40. J. S. Damoiseaux, R. P. Viviano, P. Yuan, N. Raz, Differential effect of age on posterior and anterior hippocampal functional connectivity. Neuroimage 133, 468-476 (2016).

41. A. Salami, S. Pudas, L. Nyberg, Elevated hippocampal resting-state connectivity underlies deficient neurocognitive function in aging. Proc Natl Acad Sci U S A 111, 17654-17659 (2014).

42. Y. L. Wang, T. Q. Li, Analysis of Whole-Brain Resting-State fMRI Data Using Hierarchical Clustering Approach. Plos One 8, (2013).

43. D. Tomasi, N. D. Volkow, Functional connectivity hubs in the human brain. Neuroimage 57, 908-917 (2011).

44. R. L. Buckner, J. Sepulcre, T. Talukdar, F. M. Krienen, H. Liu, T. Hedden, J. R. Andrews-Hanna, R. A. Sperling, K. A. Johnson, Cortical hubs revealed by intrinsic functional connectivity: mapping, assessment of stability, and relation to Alzheimer's disease. J Neurosci 29, 1860-1873 (2009).

45. L. Geerligs, K. A. Tsvetanov, C. Cam, R. N. Henson, Challenges in measuring individual differences in functional connectivity using fMRI: The case of healthy aging. Hum Brain Mapp 38, 4125-4156 (2017).

46. A. Hussein, J. L. Matthews, C. Syme, C. Macgowan, B. J. MacIntosh, Z. Shirzadi, Z. Pausova, T. Paus, J. J. Chen, The association between resting-state functional magnetic resonance imaging and aortic pulsewave velocity in healthy adults. Hum Brain Mapp 41, 2121-2135 (2020).

47. R. L. Buckner, J. R. Andrews-Hanna, D. L. Schacter, The brain's default network: anatomy, function, and relevance to disease. Ann N Y Acad Sci 1124, 1-38 (2008).

48. N. Luo, J. Sui, A. Abrol, D. Lin, J. Chen, V. M. Vergara, Z. Fu, Y. Du, E. Damaraju, Y. Xu, J. A. Turner, V. D. Calhoun, Age-related structural and functional variations in 5,967 individuals across the adult lifespan. Hum Brain Mapp 41, 1725-1737 (2020).

49. T. Lu, L. Aron, J. Zullo, Y. Pan, H. Kim, Y. Chen, T. H. Yang, H. M. Kim, D. Drake, X. S. Liu, D. A. Bennett, M. P. Colaiacovo, B. A. Yankner, REST and stress resistance in ageing and Alzheimer's disease. Nature 507, 448-454 (2014).

50. S. C. Williams, Alzheimer's disease: Mapping the brain's decline. Nature 502, S84-85 (2013). 
51. N. Persson, J. Wu, Q. Zhang, T. Liu, J. Shen, R. Bao, M. Ni, T. Liu, Y. Wang, P. Spincemaille, Age and sex related differences in subcortical brain iron concentrations among healthy adults. Neuroimage 122, 385398 (2015).

52. N. Persson, P. Ghisletta, C. L. Dahle, A. R. Bender, Y. Yang, P. Yuan, A. M. Daugherty, N. Raz, Regional brain shrinkage over two years: individual differences and effects of pro-inflammatory genetic polymorphisms. Neuroimage 103, 334-348 (2014).

53. W. Koch, S. Teipel, S. Mueller, K. Buerger, A. L. Bokde, H. Hampel, U. Coates, M. Reiser, T. Meindl, Effects of aging on default mode network activity in resting state $\mathrm{fMRI}$ : does the method of analysis matter? Neuroimage 51, 280-287 (2010).

54. N. Persson, P. Ghisletta, C. L. Dahle, A. R. Bender, Y. Yang, P. Yuan, A. M. Daugherty, N. Raz, Regional brain shrinkage and change in cognitive performance over two years: The bidirectional influences of the brain and cognitive reserve factors. Neuroimage 126, 15-26 (2016).

55. M. G. Bright, C. R. Tench, K. Murphy, Potential pitfalls when denoising resting state fMRI data using nuisance regression. Neuroimage 154, 159-168 (2017).

56. J. Muschelli, M. B. Nebel, B. S. Caffo, A. D. Barber, J. J. Pekar, S. H. Mostofsky, Reduction of motionrelated artifacts in resting state $\mathrm{fMRI}$ using aCompCor. Neuroimage 96, 22-35 (2014).

57. L. Parkes, B. Fulcher, M. Yucel, A. Fornito, An evaluation of the efficacy, reliability, and sensitivity of motion correction strategies for resting-state functional MRI. Neuroimage 171, 415-436 (2018).

58. R. H. R. Pruim, M. Mennes, D. van Rooij, A. Llera, J. K. Buitelaar, C. F. Beckmann, ICA-AROMA: A robust ICA-based strategy for removing motion artifacts from fMRI data. Neuroimage 112, 267-277 (2015).

59. T. Wu, Y. Zang, L. Wang, X. Long, M. Hallett, Y. Chen, K. Li, P. Chan, Aging influence on functional connectivity of the motor network in the resting state. Neurosci Lett 422, 164-168 (2007).

60. A. M. Fjell, L. McEvoy, D. Holland, A. M. Dale, K. B. Walhovd, I. Alzheimer's Disease Neuroimaging, What is normal in normal aging? Effects of aging, amyloid and Alzheimer's disease on the cerebral cortex and the hippocampus. Prog Neurobiol 117, 20-40 (2014).

61. K. K. Ng, J. C. Lo, J. K. W. Lim, M. W. L. Chee, J. Zhou, Reduced functional segregation between the default mode network and the executive control network in healthy older adults: A longitudinal study. Neuroimage 133, 321-330 (2016).

62. A. M. Staffaroni, J. A. Brown, K. B. Casaletto, F. M. Elahi, J. Deng, J. Neuhaus, Y. Cobigo, P. S. Mumford, S. Walters, R. Saloner, A. Karydas, G. Coppola, H. J. Rosen, B. L. Miller, W. W. Seeley, J. H. Kramer, The Longitudinal Trajectory of Default Mode Network Connectivity in Healthy Older Adults Varies As a Function of Age and Is Associated with Changes in Episodic Memory and Processing Speed. J Neurosci 38, 2809-2817 (2018).

63. Q. Li, C. Dong, T. Liu, X. Chen, A. Perry, J. Jiang, J. Cheng, H. Niu, N. A. Kochan, H. Brodaty, P. S. Sachdev, W. Wen, Longitudinal Changes in Whole-Brain Functional Connectivity Strength Patterns and the Relationship With the Global Cognitive Decline in Older Adults. Front Aging Neurosci 12, 71 (2020).

64. J. E. Chen, L. D. Lewis, C. Chang, Q. Tian, N. E. Fultz, N. A. Ohringer, B. R. Rosen, J. R. Polimeni, Restingstate "physiological networks". Neuroimage 213, 116707 (2020).

65. R. N. Spreng, W. D. Stevens, J. D. Viviano, D. L. Schacter, Attenuated anticorrelation between the default and dorsal attention networks with aging: evidence from task and rest. Neurobiol Aging 45, 149-160 (2016).

66. Y. Liu, L. Huang, M. Li, Z. Zhou, D. Hu, Anticorrelated networks in resting-state fMRI-BOLD data. Biomed Mater Eng 26 Suppl 1, S1201-1211 (2015).

67. K. Gopinath, V. Krishnamurthy, R. Cabanban, B. A. Crosson, Hubs of Anticorrelation in High-Resolution Resting-State Functional Connectivity Network Architecture. Brain Connect 5, 267-275 (2015).

68. J. Gonzalez-Castillo, Z. S. Saad, D. A. Handwerker, S. J. Inati, N. Brenowitz, P. A. Bandettini, Whole-brain, time-locked activation with simple tasks revealed using massive averaging and model-free analysis. Proc Natl Acad Sci U S A 109, 5487-5492 (2012). 
69. A. J. Schwarz, J. McGonigle, Negative edges and soft thresholding in complex network analysis of resting state functional connectivity data. Neuroimage 55, 1132-1146 (2011).

70. M. Bianciardi, M. Fukunaga, P. van Gelderen, J. A. de Zwart, J. H. Duyn, Negative BOLD-fMRI signals in large cerebral veins. J Cereb Blood Flow Metab 31, 401-412 (2011).

71. A. Weissenbacher, C. Kasess, F. Gerstl, R. Lanzenberger, E. Moser, C. Windischberger, Correlations and anticorrelations in resting-state functional connectivity MRI: a quantitative comparison of preprocessing strategies. Neuroimage 47, 1408-1416 (2009).

72. M. D. Fox, D. Zhang, A. Z. Snyder, M. E. Raichle, The global signal and observed anticorrelated resting state brain networks. J Neurophysiol 101, 3270-3283 (2009).

73. M. Gruszecki, G. Lancaster, A. Stefanovska, J. P. Neary, R. T. Dech, W. Guminski, A. F. Frydrychowski, J. Kot, P. J. Winklewski, Human subarachnoid space width oscillations in the resting state. Sci Rep 8, 3057 (2018).

74. M. Li, Y. Gao, F. Gao, A. W. Anderson, Z. Ding, J. C. Gore, Functional engagement of white matter in resting-state brain networks. Neuroimage 220, 117096 (2020).

75. T.-L. Wu, F. Wang, M. Li, K. G. Schilling, Y. Gao, A. W. Anderson, L. M. Chen, Z. Ding, J. C. Gore, Restingstate white matter-cortical connectivity in non-human primate brain. Neurolmage 184, $45-55$ (2019).

76. J. C. Gore, M. Li, Y. Gao, T. L. Wu, K. G. Schilling, Y. Huang, A. Mishra, A. T. Newton, B. P. Rogers, L. M. Chen, A. W. Anderson, Z. Ding, Functional MRI and resting state connectivity in white matter - a minireview. Magn Reson Imaging 63, 1-11 (2019).

77. P. McColgan, S. Gregory, A. Razi, K. K. Seunarine, F. Gargouri, A. Durr, R. A. Roos, B. R. Leavitt, R. I. Scahill, C. A. Clark, S. J. Tabrizi, G. Rees, H. D. I. Track On, A. Coleman, J. Decolongon, M. Fan, T. Petkau, C. Jauffret, D. Justo, S. Lehericy, K. Nigaud, R. Valabregue, A. Choonderbeek, E. P. Hart, D. J. Hensman Moss, H. Crawford, E. Johnson, M. Papoutsi, C. Berna, R. Reilmann, N. Weber, J. Stout, I. Labuschagne, B. Landwehrmeyer, $\mathrm{M}$. Orth, $\mathrm{H}$. Johnson, White matter predicts functional connectivity in premanifest Huntington's disease. Ann Clin Transl Neurol 4, 106-118 (2017).

78. L. Sakka, G. Coll, J. Chazal, Anatomy and physiology of cerebrospinal fluid. Eur Ann Otorhinolaryngol Head Neck Dis 128, 309-316 (2011). 
bioRxiv preprint doi: https://doi.org/10.1101/2021.02.04.429600; this version posted February 5, 2021. The copyright holder for this preprint (which was not certified by peer review) is the author/funder. All rights reserved. No reuse allowed without permission.

Table 1: The MNI coordinates for the 4 voxels of different tissue types illustrated in Fig. 5.

\begin{tabular}{cccc}
\hline Tissue & $\mathrm{X}_{\mathrm{cm}}$ & $\mathrm{Y}_{\mathrm{cm}}$ & $\mathrm{Z}_{\mathrm{cm}}$ \\
\hline CSF & +26 & +48 & +8 \\
WM & -22 & -16 & +43 \\
MC & -56 & -12 & +43 \\
PCC & 0 & +56 & +26 \\
\hline
\end{tabular}


Table 2: The brain regions where the connectivity strength metrics are significantly $(\mathrm{p}<0.05)$ correlated with the subjects' ages. The volume, center of mass coordinates in MNI space, regression parameter $(\beta)$, linear correlation coefficient $(r)$, statistical significance $(p)$, and anatomic annotations are specified. The default is bilateral, while $\mathrm{R}$ and L- indicate the right and left hemisphere of the brain, respectively. $\mathrm{CSI}_{\mathrm{NP}}$ indicates the overlapping results between $\mathrm{CSI}_{\mathrm{N}}$ and $\mathrm{CSI}_{\mathrm{P}}$.

\begin{tabular}{|c|c|c|c|c|c|c|c|c|}
\hline RFC & voxel & $X_{c m}$ & $Y_{c m}$ & $Z_{c m}$ & $\beta\left(10^{3}\right)$ & $r$ & $p\left(10^{-3}\right)$ & Annotation \\
\hline \multirow{3}{*}{ CSI } & 239 & +2.1 & +63.0 & +47.9 & 9.50 & 0.459 & $<0.01$ & precuneus \\
\hline & 152 & +3.4 & -47.3 & +30.1 & -9.72 & -0.389 & $<0.01$ & Superior and MFG \\
\hline & 62 & -38.5 & +55.3 & -35.5 & 9.57 & 0.354 & $<0.01$ & R-IPL \\
\hline \multirow{9}{*}{$\mathrm{CSI}_{\mathrm{N}}$} & 237 & +0.0 & +53.1 & +27.3 & 12.29 & 0.413 & $<0.01$ & PCC \\
\hline & 171 & -42.6 & -10.4 & +11.0 & 12.36 & 0.411 & $<0.01$ & R-insula cortex \\
\hline & 161 & -2.3 & +28.2 & +59.4 & -11.05 & -0.371 & $<0.01$ & paracentral lobule \\
\hline & 153 & -49.1 & +47.6 & +41.2 & 11.62 & 0.441 & $<0.01$ & R- IPL \\
\hline & 133 & -39.8 & +25.7 & +55.5 & -10.99 & -0.325 & $<0.01$ & R-postcentral gyrus \\
\hline & 75 & -27.3 & -3.8 & -34.0 & -9.99 & -0.450 & $<0.01$ & R-PHC \\
\hline & 67 & -56.8 & +14.6 & +5.9 & -9.28 & -0.400 & $<0.01$ & R-STG \\
\hline & 58 & +18.4 & +0.4 & -18.9 & -9.36 & -0.441 & $<0.01$ & L-PHC \\
\hline & 56 & +42.0 & +25.9 & +57.1 & -10.38 & -0.309 & $<0.01$ & L-postcentral gyrus \\
\hline \multirow{6}{*}{$\mathrm{CSI}_{\mathrm{p}}$} & 713 & +2.0 & -45.4 & +23.6 & -10.37 & -0.487 & $<0.01$ & Superior and MFG \\
\hline & 157 & -1.1 & +14.7 & -17.8 & 7.96 & 0.506 & $<0.01$ & putamen \\
\hline & 110 & +55.7 & +13.1 & -19.5 & -9.49 & -0.433 & $<0.01$ & L-MTG \\
\hline & 75 & +2.7 & +48.5 & +31.1 & -9.01 & -0.336 & $<0.01$ & PCC \\
\hline & 53 & -40.0 & -8.7 & +0.8 & -8.48 & -0.361 & $<0.01$ & R-insula cortex \\
\hline & 52 & +45.1 & -10.5 & -8.9 & -8.36 & -0.376 & $<0.01$ & L-insula cortex \\
\hline \multirow{4}{*}{$\mathrm{CSI}_{\mathrm{N}} \cap \mathrm{CSI} \mathrm{P}_{\mathrm{P}}$} & 70 & +2.8 & +49.0 & +31.0 & 14.15 & 0.374 & $<0.01$ & $\operatorname{PCC}\left(\mathrm{CSI}_{N}\right)$ \\
\hline & & & & & -9.04 & -0.334 & $<0.01$ & $\mathrm{PCC}\left(\mathrm{CSI} \mathrm{P}_{\mathrm{P}}\right)$ \\
\hline & 34 & -40.8 & -10.0 & +0.0 & 13.37 & 0.362 & $<0.01$ & R-Insula cortex $\left(\mathrm{CSI}_{N}\right)$ \\
\hline & & & & & -8.63 & -0.336 & $<0.01$ & R-Insula cortex $\left(\mathrm{CSI}_{\mathrm{P}}\right)$ \\
\hline
\end{tabular}


Table 3: The joint overlapping brain regions where the connectivity density medics of different kernels are all significantly $(\mathrm{p}<0.05)$ correlated with the subjects' ages. The volume, center of mass coordinates in MNI space, and anatomic annotations, regression parameters $(\beta)$, linear correlation coefficient $(r)$, statistical significance $(p)$, and anatomic annotations are specified. The default is bilateral, while R and L- indicate the right and left hemisphere of the brain, respectively. $\cap C D I_{N}$ and nCDIP indicate the joint overlaps among the $\mathrm{CDI}_{N}$ and $C D I_{P}$ metrics of the different kernels, respectively. The $\beta, r$ and $p$ are the average results for the 6 different kernels.

\begin{tabular}{|c|c|c|c|c|c|c|c|c|}
\hline RFC & voxel & $\mathrm{X}_{\mathrm{cm}}$ & $\mathrm{Y}_{\mathrm{cm}}$ & $Z_{\mathrm{cm}}$ & $\beta\left(10^{3}\right)$ & $r$ & $p\left(10^{-3}\right)$ & Notations \\
\hline \multirow{10}{*}{$\cap C D I_{N}$} & 216 & +0.1 & +51.9 & +29.2 & -13.81 & -0.376 & $<0.01$ & PCC \\
\hline & 103 & +0.5 & +28.2 & +57.6 & 12.16 & 0.366 & $<0.01$ & paracentral lobule \\
\hline & 92 & -48.2 & +47.0 & +42.1 & -13.32 & -0.431 & $<0.01$ & R-IPL \\
\hline & 72 & -38.7 & +26.2 & +57.2 & 11.91 & 0.326 & $<0.01$ & R-post central gyrus \\
\hline & 44 & +21.2 & -4.7 & -20.6 & 9.95 & 0.430 & $<0.01$ & L-PHC \\
\hline & 38 & -41.9 & -12.4 & +5.0 & -15.16 & -0.374 & $<0.01$ & R-insula cortex \\
\hline & 29 & +20.8 & -4.0 & -20.0 & 8.75 & 0.371 & $<0.01$ & L-STG \\
\hline & 29 & -1.5 & -14.4 & +39.5 & -11.66 & -0.355 & $<0.01$ & Anterior cingulate cortex \\
\hline & 28 & +44.0 & +24.1 & +57.1 & 10.37 & 0.297 & $<0.01$ & L-post central gyrus \\
\hline & 20 & -45.2 & +18.4 & +9.6 & 9.20 & 0.364 & $<0.01$ & R-STG \\
\hline \multirow{3}{*}{ nCDIp } & 567 & +4.2 & -46.9 & +24.8 & -12.20 & -0.480 & $<0.01$ & Superior and MFG \\
\hline & 136 & -9.4 & +21.2 & -22.3 & 8.53 & 0.452 & $<0.01$ & Putamen \\
\hline & 30 & +57.0 & +15.8 & -15.8 & -10.09 & -0.387 & $<0.01$ & L-MTG \\
\hline
\end{tabular}


Table 4. The linear regression results for the 3 ROIs with significant correlation between CSI and the subject's age. The CSIP and CSIN results are based on the masks determined solely by the CSI results.

\begin{tabular}{l|rr|rr|rr}
\hline \multirow{2}{*}{ ROI } & \multicolumn{2}{|c|}{$\mathrm{CSI}_{\mathrm{P}}$} & \multicolumn{2}{c|}{$\mathrm{CSI}_{\mathrm{N}}$} & \multicolumn{2}{c}{$\mathrm{CSI}$} \\
& \multicolumn{1}{|c|}{$\beta \times 10^{3}$} & \multicolumn{1}{c|}{$\beta \times 10^{3}$} & $r$ & $\beta \times 10^{3}$ & $r$ \\
\hline precuneus & 4.93 & 0.283 & 3.17 & 0.175 & 9.50 & 0.459 \\
MFG & -11.46 & -0.441 & 3.77 & 0.136 & -9.72 & -0.389 \\
R-IPL & 0.73 & 0.031 & 8.03 & 0.299 & 9.57 & 0.354 \\
\hline
\end{tabular}




\section{Figure Captions}

Fig. 1: A schematic overview to illustrate the QDA framework. With QDA the time course of each voxel is used in turn to compute the whole-brain CC matrix. For each row of the CC matrix, we compute a CC histogram with 200 evenly binned intervals within [-1, 1]. The histogram shown in the graph is the cohort's average CC histogram for a voxel within the PCC, whose location was marked with the red square. In QDA, two types of RFC images are derived from the CC matrix: 1) CSI $I_{P}$ and $\mathrm{CSI}_{N}$ whose voxel values are the averages of the positives and negatives in each row of the CC matrix, respectively. 2) $\mathrm{CDI}_{\mathrm{P}}$ and $\mathrm{CDI}_{\mathrm{N}}$ whose voxel values are the positive and negative parts of the convolution between the CC histogram and the kernel, respectively.

Fig. 2: An axial slice of the average $\mathrm{CSI}_{N}(\mathrm{a})$ and $\mathrm{CSI}_{\mathrm{P}}(\mathrm{b})$ for the cohort before $(\mathrm{a}, \mathrm{b})$ and after the Zscore transformation $(c, d)$.

Fig. 3: The six different kernel functions investigated in the study to derive the $\mathrm{CDI}_{\mathrm{P}}$ and $\mathrm{CDI}_{\mathrm{N}}$ metrics. The widely used threshold method can be considered as the case for the square-well kernel function $\left(k_{6}\right)$.

Fig. 4: The average CC histogram of the cohort for a seed voxel in the posterior cingulate cortex (PCC) as indicated by the green cross. Selecting different threshold values along the histogram allows us to detect the functional connection networks of different strengths (a-f) associated with the seed voxel in the PCC.

Fig. 5: Anatomic locations for 4 different seed voxels of different tissue types including white matter (WM), cerebral spinal fluid (CSF), PCC and motor cortex (MC).

Fig. 6: The average CC histograms of the cohort for the 4 different seed voxels shown in Fig. 5.

Fig. 7: The convolutions of the CC histograms shown in Fig. 6 for the 4 different seed voxels located in (a) WM, (b) PCC, (c) CSF and (d) MC.

Fig. 8: An axial slice of the $\mathrm{CDI}_{\mathrm{P}}$ (upper row) and $\mathrm{CDI}_{\mathrm{N}}$ (lower row) metrics derived from a typical RfMRI dataset (a male subject of 36 years' age). The images from left to right depict the results for the following 6 kernel functions $|x|,\left|x^{2}\right|,\left|x^{3}\right|,\left|x^{4}\right|, \sin ^{2}(\pi / 2 x)$ and $\operatorname{step}(|x|-0.3)$, respectively. 
Fig. 9: The average $\mathrm{CDI}_{\mathrm{P}}(\mathrm{a})$ and $\mathrm{CDI}_{N}(\mathrm{~b})$ histograms for the cohort derived by using the 6 different kernels $k_{1}-k_{6}$.

Fig. 10: The differences in CDI histograms between the young and elderly sub-groups. The CDI and $\mathrm{CDI}_{\mathrm{P}}$ results for the 6 different kernels are shown.

Fig. 11: The $\mathrm{CDI}_{\mathrm{P}}$ and $\mathrm{CDI}_{N}$ histogram differences between the young and elderly sub-groups as a function of the kernels. Each data point corresponds to the total area above and below the '0' horizontal line of the histogram differences shown in Fig. 10.

Fig. 12: Brain regions with significant correlation $(p<0.05$, corrected) between the connectivity strength metrics and the subject's age. The results for the CSI (a), $\mathrm{CSI}_{N}(\mathrm{~b})$ and $\mathrm{CSI}_{\mathrm{P}}(\mathrm{c})$ are depicted separately. The Color bar shows the t-score level.

Fig. 13: The overlapping ROIs in the PCC and right insula cortex where both the CSIP and CSI metrics depict significant decline with the adult age (a). The average Pearson's correlation maps of the cohort associated the overlapping ROI seeds in the PCC (b) and insula cortex (c).

Fig. 14: Cross-sectional display of the anti-correlation networks associated with the 2 overlapping ROIs as derived from the product of the maps shown in Figs. $13 \mathrm{~b}$ and $13 \mathrm{c}$ at $\mathrm{CC} \leq(-0.5)$. The crossing points of green lines depict the center of mass for each ROI

Fig. 15: The ROI average of the $\mathrm{CSI}_{N}$ metric against the subject's age for the overlapping ROI in the PCC (a). The ROI average for the CSIN metric against the subject's age for the overlapping ROI in the right insula cortex (b). The ROI average for the CSI metric against the subject's age for the overlapping ROI in the PCC (c). The ROI average for the $\mathrm{CSI}_{\mathrm{P}}$ metric against the subject's age for the overlapping ROI in the insula cortex (d). The lines show the linear regression results of the RFC metrics against the subject's ages.

Fig. 16: The total volumes of the detected brain regions with significant correlation $(p<0.05$, corrected) between the connectivity density index $\left(\mathrm{CDI}_{\mathrm{P}}\right.$ and $\left.\mathrm{CDI}_{\mathrm{N}}\right)$ and the subject's age as a function of the kernels (a). The average regression parameter $\beta$ for the detected brain regions as a function of the kernels (b). The negative and positive correlations were assessed separately. 
bioRxiv preprint doi: https://doi.org/10.1101/2021.02.04.429600; this version posted February 5, 2021. The copyright holder for this preprint (which was not certified by peer review) is the author/funder. All rights reserved. No reuse allowed without permission.

Fig. 17: The ROI average of the $\mathrm{CSI}_{\mathrm{P}}, \mathrm{CSI}_{\mathrm{N}}$ and $\mathrm{CSI}$ metrics against the subject's age for the $3 \mathrm{ROIs}$ with significant correlation between CSI and the subject's age. The columns 1 to 3 are the results for the ROls in the precuneus, MFG, and R-IPL, respectively. The rows 1 to 3 are the results for the $\mathrm{CSI}_{\mathrm{P}}, \mathrm{CSI}_{\mathrm{N}}$ and CSI metrics, respectively. The ROI masks are solely based on the CSI metric only. 